\title{
ULRICH IDEALS AND MODULES OVER TWO-DIMENSIONAL RATIONAL SINGULARITIES
}

\author{
SHIRO GOTO, KAZUHO OZEKI, RYO TAKAHASHI, \\ KEI-ICHI WATANABE AND KEN-ICHI YOSHIDA
}

\begin{abstract}
The main aim of this paper is to classify Ulrich ideals and Ulrich modules over two-dimensional Gorenstein rational singularities (rational double points) from a geometric point of view. To achieve this purpose, we introduce the notion of (weakly) special Cohen-Macaulay modules with respect to ideals, and study the relationship between those modules and Ulrich modules with respect to good ideals.
\end{abstract}

\section{CONTENTS}

1 Introduction 69

2 Preliminaries 73

3 Weakly special Cohen-Macaulay modules over Gorenstein local domains 77

4 Special Cohen-Macaulay modules over two-dimensional rational singularities $\quad 80$

5 Ulrich ideals and modules over rational double points 86

6 Ulrich ideals of non-Gorenstein rational singularities $\quad 98$

7 Examples 104

References 108

\section{$\S 1$. Introduction}

In the paper [GOTWY] we established the theory of Ulrich ideals and modules with a generalized form. The classical concept of Ulrich modules, or maximally generated maximal Cohen-Macaulay modules (MGMCM modules), was introduced by [U, BHU]. In our language, MGMCM modules

Received November 30, 2013. Revised October 2, 2014. Accepted October 6, 2014. 2010 Mathematics subject classification. Primary 13C14, 14B05, 14C25, 14E16.

This work was partially supported by JSPS Grant-in-Aid for Scientific Research (C) $20540050 / 22540047 / 22540054 / 23540059$, by JSPS Grant-in-Aid for Young Scientists (B) 22740008/22740026 and by JSPS Postdoctoral Fellowships for Research Abroad.

(C) 2016 by The Editorial Board of the Nagoya Mathematical Journal 
are just Ulrich modules with respect to the maximal ideal. For instance, any indecomposable nonfree maximal Cohen-Macaulay module over a hypersurface local ring of multiplicity (degree) 2 is an MGMCM module. While they are very few MGMCM modules in general, our Ulrich modules include many more members than MGMCM modules.

To state the main results, let us begin with the definition of Ulrich ideals and modules. Let $A$ be a Cohen-Macaulay local ring with maximal ideal $\mathfrak{m}$ and $d=\operatorname{dim} A \geqslant 0$, and let $I \subset A$ be an $\mathfrak{m}$-primary ideal. For simplicity, we assume that $I$ contains a parameter ideal $Q=\left(a_{1}, a_{2}, \ldots, a_{d}\right)$ of $A$ as a reduction, that is, $I^{r+1}=Q I^{r}$ for some integer $r \geqslant 1$.

Definition 1.1. We say that $I$ is an Ulrich ideal of $A$ if it satisfies the following conditions:

(1) $I^{2}=Q I$

(2) $I / I^{2}$ is a free $A / I$-module.

Let $\mathcal{X}_{A}$ denote the set of all Ulrich ideals that are not parameter ideals.

For instance, $(A, \mathfrak{m})$ is a Cohen-Macaulay local ring of maximal embedding dimension $[\mathrm{Sa}]$ if and only if $\mathfrak{m}$ is an Ulrich ideal.

Definition 1.2. Let $M$ be a nonzero finitely generated $A$-module, and let $I \subset A$ be an $\mathfrak{m}$-primary ideal. Then we say that $M$ is an Ulrich $A$-module with respect to $I$, if the following conditions are satisfied:

(1) $M$ is a maximal Cohen-Macaulay $A$-module;

(2) $\mathrm{e}_{I}^{0}(M)=\ell_{A}(M / I M)$;

(3) $M / I M$ is $A / I$-free.

Here, $\mathrm{e}_{I}^{0}(M)$ denotes the multiplicity of $M$ with respect to $I$ and $\ell_{A}(M / I M)$ denotes the length of the $A$-module $M / I M$.

In [GOTWY], we proved that all higher syzygy modules $\operatorname{Syz}_{A}^{i}(A / I)$ of an Ulrich ideal $I$ are Ulrich modules with respect to $I$. Moreover, if $A$ is of finite Cohen Macaulay $(\mathrm{CM})$ representation type, then $\mathcal{X}_{A}$ is a finite set. Recall here that a Cohen-Macaulay local ring is said to be of finite CMrepresentation type if there are only a finite number of isomorphism classes of indecomposable maximal Cohen-Macaulay $A$-modules. Thus, we consider the following natural question. 
Problem 1.3. Let $(A, \mathfrak{m})$ be a Cohen-Macaulay local ring of finite CMrepresentation type.

(1) Classify all Ulrich ideals $I$ of $A$.

(2) Classify all Ulrich $A$-modules with respect to a given $\mathfrak{m}$-primary ideal $I$.

(3) Determine all $\mathfrak{m}$-primary ideals $I$ so that there exists an Ulrich $A$ module with respect to $I$.

In [GOTWY, Section 9], we gave an answer to the problem as above in the case of a one-dimensional Gorenstein local ring of finite CM-representation type by using techniques from representation theory of maximal CohenMacaulay modules. We want to give a complete answer to the question as above in the case of a two-dimensional Gorenstein local ring of finite CMrepresentation type. It is well known that two-dimensional Gorenstein local rings of finite CM-representation type (over an algebraically closed field of characteristic 0) are two-dimensional Gorenstein rational singularities.

Let us explain the organization of the paper. In Section 3, we introduce the notion of weakly special Cohen-Macaulay modules; let $A$ be a CohenMacaulay local domain, and let $I \subset A$ be an $\mathfrak{m}$-primary ideal. A maximal Cohen-Macaulay $A$-module $M$ is called a weakly special Cohen-Macaulay $A$-module with respect to $I$ if $\mu_{A}(M)=2 \cdot \operatorname{rank}_{A} M$ and $M / I M$ is $A / I$ free, where $\mu_{A}(M)$ denotes the cardinality of a minimal set of generators of $M$; see Definition 3.1. Then we prove that $M$ is an Ulrich $A$-module with respect to $I$ and $I$ is a good ideal (see Section 2) if and only if $M$ is a weakly special Cohen-Macaulay $A$-module with respect to $I$ for a Gorenstein local domain $A$ and a nonparameter m-primary stable ideal $I$; see Theorem 3.2 for details. As an application, we give a partial answer to the Problem 1.3(3). This implies that $I$ is an Ulrich ideal if and only if there exists an Ulrich $A$-module with respect to $I$ for any two-dimensional Gorenstein rational singularity.

In Section 4, we modify the notion of special Cohen-Macaulay $A$ modules introduced by Wunram [Wu1] (see also [Ito, IW]). Let $A$ be a twodimensional rational singularity, and let $M$ be a maximal Cohen-Macaulay $A$-module without free summands. Then $M$ is a special Cohen-Macaulay $A$-module with respect to $I$ if and only if $\operatorname{Ext}_{A}^{1}(M, A)=0$ and $M / I M$ is $A / I$-free; see Definition 4.5. Special Cohen-Macaulay $A$-modules are weakly 
special Cohen-Macaulay $A$-modules (but the converse is not true in general). The main result in this section is the following theorem, which gives a criterion for $I$ (resp. $Z$ ) to be a special ideal (resp. a special cycle) in terms of cycles.

THEOREM 4.10. Let $(A, \mathfrak{m})$ be a two-dimensional complete local normal domain with rational singularity having the minimal resolution of singularities with the exceptional divisor $E=\bigcup_{i=1}^{r} E_{j}$. Let $Z=\sum_{j=1}^{r} a_{j} E_{j} \neq Z_{0}$ be an anti-nef cycle on the minimal resolution $X \rightarrow \operatorname{Spec} A$, and put $I=I_{Z}$. Let $Z_{0}=\sum_{j=1}^{r} n_{j} E_{j}$ denote the fundamental cycle on $X$. Let $M_{i}$ be an indecomposable maximal Cohen-Macaulay A-module so that $c_{1}\left(\widetilde{M}_{i}\right) E_{j}=$ $\delta_{i, j}, 1 \leqslant i, j \leqslant r$; see Theorem-Definition 4 for details. Then the following conditions are equivalent for every $i, 1 \leqslant i \leqslant r$.

(1) $M_{i}$ is a special Cohen-Macaulay A-module with respect to $I$.

(2) $a_{i}=n_{i} \cdot \ell_{A}(A / I)$.

(3) There exist positive cycles $0<Y_{s} \leqslant \cdots \leqslant Y_{1} \leqslant Z_{0}$ and anti-nef cycles $Z_{1}, \ldots, Z_{s}$ so that $Z_{k}=Z_{k-1}+Y_{k}$ for each $k=1, \ldots, s$ and

$$
\begin{aligned}
& Z_{k-1} \cdot Y_{k}=0, \quad p_{a}\left(Y_{k}\right)=0 \text { and } \operatorname{coeff}_{E_{i}} Y_{k}=n_{i} \\
& \quad \text { for every } k=1,2, \ldots, s,
\end{aligned}
$$

where coeff $E_{i} W$ stands for the coefficient of $E_{i}$ in a cycle $W$.

When this is the case, $\ell_{A}(A / I)=s+1$ and every $I_{k}:=I_{Z_{k}}$ is a special ideal. Moreover, for every $k=1,2, \ldots, s$, we obtain that $\operatorname{Supp}\left(Y_{k}\right)$ is connected, $\operatorname{Supp}\left(Y_{k}\right) \subset \cup\left\{E_{j} \subset \operatorname{Supp}\left(Y_{k-1}\right) \mid E_{j} Z_{k-1}=0\right\}$, and $Y_{k}$ is the fundamental cycle on $\operatorname{Supp}\left(Y_{k}\right)$.

In Section 5, we give a complete list of Ulrich ideals and Ulrich modules with respect to some ideal $I$ for any two-dimensional Gorenstein rational Cohen-Macaulay singularity. The main tools are the Riemann-Roch formula, the McKay correspondence and results in Section 4. The following theorem is the main result in this paper.

THEOREM 1.4. Let $A$ be a two-dimensional Gorenstein rational singularity. Then the set $\mathcal{X}_{A}$ of all nonparameter Ulrich ideals is given by the following. 


$$
\begin{aligned}
\left(A_{2 m}\right) \quad & \left\{(x, y, z),\left(x, y^{2}, z\right), \ldots,\left(x, y^{m}, z\right)\right\} . \\
\left(A_{2 m+1}\right) \quad & \left\{(x, y, z),\left(x, y^{2}, z\right), \ldots,\left(x, y^{m+1}, z\right)\right\} . \\
\left(D_{2 m}\right) \quad\left\{(x, y, z),\left(x, y^{2}, z\right), \ldots,\left(x, y^{m-1}, z\right)\right. & \left.\left(x+\sqrt{-1} y^{m-1}, y^{m}, z\right),\left(x-\sqrt{-1} y^{m-1}, y^{m}, z\right),\left(x^{2}, y, z\right)\right\} . \\
\left(D_{2 m+1}\right) & \left\{(x, y, z),\left(x, y^{2}, z\right), \ldots,\left(x, y^{m}, z\right),\left(x^{2}, y, z\right)\right\} . \\
\left(E_{6}\right) \quad & \left\{(x, y, z),\left(x, y^{2}, z\right)\right\} . \\
\left(E_{7}\right) \quad & \left\{(x, y, z),\left(x, y^{2}, z\right),\left(x, y^{3}, z\right)\right\} . \\
\left(E_{8}\right) & \left\{(x, y, z),\left(x, y^{2}, z\right)\right\} .
\end{aligned}
$$

In Section 6, we discuss Ulrich ideals of two-dimensional non-Gorenstein rational singularities. We show that any Ulrich ideal is integrally closed and is represented on the minimal resolution of singularities, and also is a special ideal in the sense of Section 4. For instance, any non-Gorenstein cyclic quotient singularity admits a unique Ulrich ideal, that is, the maximal ideal (see also Section 7).

\section{$\S 2$. Preliminaries}

\subsection{Ulrich ideals and modules}

First we recall the notion of good ideals in a Gorenstein local ring.

Definition 2.1. (See [GIW].) Suppose that $A$ is a Gorenstein local ring. Let $I \subset A$ be a nonparameter $\mathfrak{m}$-primary ideal. If $I^{2}=Q I$ holds for some minimal reduction $Q$ of $I$, then $I$ is called a stable ideal. If $I$ is stable and $Q: I=I$, then $I$ is called a good ideal. An $\mathfrak{m}$-primary stable ideal $I$ is good if and only if $e_{I}^{0}(A)=2 \cdot \ell_{A}(A / I)$.

An Ulrich ideal in a Gorenstein local ring is always a good ideal.

Proposition 2.2. (See [GOTWY, Lemma 2.3, Corollary 2.6].) Let $A$ be a d-dimensional Cohen-Macaulay local ring, and let $I \subset A$ be a nonparameter $\mathfrak{m}$-primary ideal. Then we have the following.

(1) Suppose that $I$ is stable. Then $e_{I}^{0}(A) \leqslant\left(\mu_{A}(I)-d+1\right) \cdot \ell_{A}(A / I)$. Equality holds if and only if $I$ is an Ulrich ideal.

(2) Suppose that $A$ is Gorenstein. Then the following conditions are equivalent: 
(a) I is an Ulrich ideal;

(b) $I$ is a good ideal and $\mu_{A}(I)=d+1$;

(c) $I$ is a good ideal and $A / I$ is Gorenstein.

Let us give two typical examples of Ulrich ideals.

EXAMPLE 2.3. It is well known that $\mu_{A}(\mathfrak{m}) \leqslant e_{\mathfrak{m}}^{0}(A)+\operatorname{dim} A-1$ holds true $[\mathrm{Ab}]$. Equality holds if and only if the maximal ideal $\mathfrak{m}$ is stable (see [Sa]). Then $A$ is said to have maximal embedding dimension. By 2.2(1), $\mathfrak{m}$ is an Ulrich ideal if and only if $A$ has maximal embedding dimension.

Suppose that $A$ is a two-dimensional hypersurface of degree 2 . Then the maximal ideal $\mathfrak{m}$ is an Ulrich ideal. Moreover, a power $\mathfrak{m}^{k}$ is a good ideal but not an Ulrich ideal for all $k \geqslant 2$.

EXAMPLE 2.4. Let $A=k\left[\left[x_{0}, x_{1}, \ldots, x_{d}\right]\right] /\left(x_{0}^{n_{0}}+\cdots+x_{d}^{n_{d}}\right)$ be a diagonal hypersurface. Suppose that $n_{0}=2 m$ is even. Then $\left(x_{0}^{m}, x_{1}^{k_{1}}, \ldots, x_{d}^{k_{d}}\right)$ is an Ulrich ideal for every $1 \leqslant k_{i} \leqslant\left\lfloor\frac{n_{i}}{2}\right\rfloor(i=1,2, \ldots, d)$.

The following theorem gives a relationship between Ulrich ideals and Ulrich modules with respect to ideals.

Theorem 2.5. (Cf. [GOTWY, Theorem 4.1].) Let $A$ be a CohenMacaulay local ring of dimension $d$. Then the following conditions are equivalent:

(1) I is a nonparameter Ulrich ideal;

(2) $\operatorname{Syz}_{A}^{i}(A / I)$ is an Ulrich $A$-module with respect to I for all $i \geqslant d$.

Note that there exists a non-Ulrich ideal $I$ so that $\operatorname{Syz}_{A}^{i}(A / I)$ is an Ulrich $A$-module with respect to $I$ (see, e.g., Examples $3.7,3.8$ ).

On the other hand, we can construct new Ulrich modules from a given Ulrich module by the following theorem.

Theorem 2.6. (See also [GOTWY, Lemma 4.2, Theorem 5.1].)

Suppose that $A$ is a Cohen-Macaulay local ring of dimension $d$ which admits a canonical module $K_{A}$. Assume that $I$ is an Ulrich ideal with $\mu(I)>d$ and $M$ is an Ulrich $A$-module with respect to $I$. Then:

(1) $\operatorname{Syz}_{A}^{1}(M)$ is an Ulrich A-module with respect to I;

(2) $M^{\vee}=\operatorname{Hom}_{A}\left(M, K_{A}\right)$ is an Ulrich A-module with respect to $I$. 


\subsection{Two-dimensional rational singularities}

Throughout this subsection, let $A$ be a two-dimensional complete normal local domain with unique maximal ideal $\mathfrak{m}$ containing an algebraically closed field $k$ of characteristic 0, unless otherwise specified. (Many results in this paper hold true if $k$ is an algebraically closed field of positive characteristic. For simplicity, we assume that $k$ has characteristic 0.) Moreover, assume that $A$ has a rational singularity, that is, there exists a resolution of singularities $\varphi: X \rightarrow \operatorname{Spec} A$ with $\mathrm{H}^{1}\left(X, \mathcal{O}_{X}\right)=0$ (see [Li1, Li2]). A typical example of rational singularities is a quotient singularity. Moreover, (two-dimensional) Gorenstein rational singularities are called rational double points, which are hypersurfaces of degree 2 .

Positive cycles, anti-nef cycles. In what follows, let $\varphi: X \rightarrow \operatorname{Spec} A$ be a resolution of singularities with $E=\varphi^{-1}(\mathfrak{m})$ the exceptional divisor. Let $E=\bigcup_{i=1}^{r} E_{i}$ be the decomposition into irreducible components of $E$. In the set $\mathcal{C}=\sum_{i=1}^{r} \mathbb{Z} E_{i}$ of cycles supported on $E$, we define a partial order $\leqslant$ as follows: for $Z, Z^{\prime} \in \mathcal{C}, Z \leqslant Z^{\prime}$ if every coefficient of $E_{i}$ in $Z^{\prime}-Z$ is nonnegative. A cycle $Z=\sum_{i=1}^{r} a_{i} E_{i}$ is called positive, denoted by $Z>0$, if $0 \leqslant Z$ and $Z \neq 0$.

On the other hand, a positive cycle $Z=\sum_{i=1} a_{i} E_{i}$ is said to be anti-nef if $Z E_{i} \leqslant 0$ for every $i=1, \ldots, r$, where $Z Y$ denotes the intersection number of $Z$ and $Y$.

Virtual genus. Since the intersection matrix $\left[E_{i} E_{j}\right]_{1 \leqslant i, j \leqslant r}$ is negative definite, there exists the unique $\mathbb{Q}$-divisor $\mathrm{K}_{X}$, the canonical divisor, so that the equation

$$
p_{a}\left(E_{i}\right):=\frac{E_{i}^{2}+\mathrm{K}_{X} E_{i}}{2}+1=0
$$

holds for every $i=1, \ldots, r$. If $E_{i}^{2}=\mathrm{K}_{X} E_{i}=-1$, then $E_{i} \cong \mathbb{P}^{1}$ is called a $(-1)$-curve. We say that $X$ is a minimal resolution if $X$ contains no (-1)curve. Such a resolution is unique up to isomorphism. Moreover, for any positive cycle $Y>0$, we put

$$
p_{a}(Y)=\frac{Y^{2}+K_{X} Y}{2}+1
$$

which is called the virtual genus of $Y$. One can easily see that

$$
p_{a}\left(Y+Y^{\prime}\right)=p_{a}(Y)+p_{a}\left(Y^{\prime}\right)+Y Y^{\prime}-1
$$


Furthermore, it is well known that if $A$ is a rational singularity then $p_{a}(Z) \leqslant$ 0 holds true for every positive cycle $Z$ [Ar, Proposition 1].

Dual graph. In what follows, assume that $\varphi: X \rightarrow \operatorname{Spec} A$ is the minimal resolution of singularities with $\varphi^{-1}(\mathfrak{m})=\bigcup_{i=1}^{r} E_{i}$. Then the dual graph $\Gamma$ of $\varphi$ is a simple graph with the vertex set $\left\{E_{i}\right\}_{i=1}^{r}$ and the edge defined by the following:

the edge $E_{i}-E_{j}$ exists (resp. does not exist) if and only if

$$
E_{i} E_{j}=1\left(\operatorname{resp} . E_{i} E_{j}=0\right) \text {. }
$$

For instance, we have the following example:

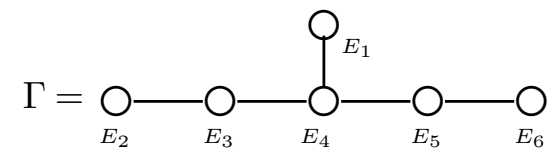

$$
\left(E_{1} E_{4}=E_{2} E_{3}=E_{3} E_{4}=E_{4} E_{5}=E_{5} E_{6}=1, \quad E_{i} E_{j}=0\right. \text { (others).) }
$$

Let $Y=\sum_{j=1}^{r} a_{j} E_{j}$ be a positive cycle on $X$. Then we put $\operatorname{Supp}(Y)=$ $\cup\left\{E_{i} \mid a_{i}>0\right\}$, the support of $Y$. Such a set is called connected if the induced subgraph is connected. Note that if $Y$ is positive and $p_{a}(Y)=0$ then $Y$ is connected.

Integrally closed ideal. Let $I$ be an m-primary ideal of $A$. An element $z \in A$ is said to be integral over $I$ if an equation

$$
z^{n}+c_{1} z^{n-1}+\cdots+c^{n}=0
$$

holds for some positive integer $n$ and $c_{i} \in I^{i}$ for each $i=1,2, \ldots, n$. The set of all elements $z$ that are integral over $I$ forms an ideal containing $I$. This ideal is called the integral closure of $I$ denoted by $\bar{I}$. An ideal $I$ is said to be integrally closed if $\bar{I}=I$.

Now suppose that $I$ is integrally closed. Then $I$ is said to be represented on $X$ if there exists an anti-nef cycle $Z$ with support in $E$ so that $I \mathcal{O}_{X}=$ $\mathcal{O}_{X}(-Z)$ and $I=\mathrm{H}^{0}\left(X, \mathcal{O}_{X}(-Z)\right)$. Then we denote such an ideal $I$ by $I=$ $I_{Z}$. The product of two integrally closed ideals of $A$ is also integrally closed [Li1, Theorem 7.1]. There is a one-to-one correspondence between the set of integrally closed $\mathfrak{m}$-primary ideals of $A$ that are represented on $X$ and the set of anti-nef cycles $Z=\sum_{i=1}^{r} a_{i} E_{i}$ on $X$ (see, e.g., [Li1, Section 18]).

Good ideal. Now we recall the notion of good ideals of rational singularities. 
Definition 2.7. Let $I$ be an $\mathfrak{m}$-primary ideal of $A$. Then $I$ is called good if $I$ is represented on the minimal resolution of singularities.

Notice that this definition is different from that of Definition 2.1. However, for any $\mathfrak{m}$-primary ideal $I$ of a two-dimensional Gorenstein rational singularity, $I$ is good in the sense of Definition 2.1 if and only if it is good in the sense of Definition 2.7 (see also [GIW, Theorem 7.8] or [WY]).

Notice that (1), (2) can be easily seen from the definition.

Lemma 2.8. (Cf. [GIW, Section 7].) Let $A$ be a two-dimensional (not necessarily Gorenstein) rational singularity, and let $\varphi: X \rightarrow \operatorname{Spec} A$ denote the minimal resolution of singularities. Then we have the following.

(1) The minimum element (say, $Z_{0}$ ) among all non-zero anti-nef cycles on $X$ exists. This cycle $Z_{0}$ is called the fundamental cycle on $X$ which corresponds to the maximal ideal $\mathfrak{m}$. In particular, $\mathfrak{m}=\mathrm{H}^{0}\left(X, \mathcal{O}_{X}\left(-Z_{0}\right)\right)$ is a good ideal.

(2) If $I=\mathrm{H}^{0}\left(X, \mathcal{O}_{X}(-Z)\right)$ and $J=\mathrm{H}^{0}\left(X, \mathcal{O}_{X}\left(-Z^{\prime}\right)\right)$ are good ideals of $A$, then $I J=\mathrm{H}^{0}\left(X, \mathcal{O}_{X}\left(-\left(Z+Z^{\prime}\right)\right)\right)$ is also a good ideal.

(3) Suppose that $Z$ is an anti-nef cycle on $X$. If $I=\mathrm{H}^{0}\left(X, \mathcal{O}_{X}(-Z)\right)$, then $\mathrm{e}_{I}^{0}(A)=-Z^{2}$.

The colength $\ell_{A}(A / I)$ can also be determined by the anti-nef cycle $Z$ (see the Riemann-Roch formula (Lemma 4.12)).

\section{$\S 3$. Weakly special Cohen-Macaulay modules over Gorenstein local domains}

Throughout this section, let $A$ be a Gorenstein local domain, and let $I \subset A$ be a nonparameter $\mathfrak{m}$-primary ideal, unless otherwise specified. In this section, we introduce the notion of weakly special Cohen-Macaulay modules, which are closely related to Ulrich modules.

Definition 3.1. (Weakly special CM module, ideal) Let $A$ be a Cohen-Macaulay local domain, and let $M$ be a maximal Cohen-Macaulay $A$-module. If $M$ satisfies $\mu_{A}(M)=2 \cdot \operatorname{rank}_{A} M$ and $M / I M$ is $A / I$-free, then $M$ is called a weakly special Cohen-Macaulay A-module with respect to $I$.

Suppose that $I \subset A$ is a stable ideal. If there exists a weakly special Cohen-Macaulay $A$-module with respect to $I$, then $I$ is called a weakly special ideal of $A$. 
Now suppose that $A$ is a Gorenstein local ring. Let $I \subset A$ be a stable $\mathfrak{m}$ primary ideal with minimal reduction $Q$. Then as $I \subseteq Q: I$, we have $I / Q \subseteq$ $(Q: I) / Q \cong K_{A / I}$. Hence,

$$
e_{I}^{0}(A)=\ell_{A}(A / Q) \leqslant \ell_{A}(A / I)+\ell\left(K_{A / I}\right)=2 \cdot \ell_{A}(A / I),
$$

where the last equality follows from the Matlis duality theorem. Note that equality holds if and only if $I$ is a good ideal.

The following theorem is the main result in this section.

Theorem 3.2. Suppose that $A$ is a Gorenstein local domain and $I$ is a stable ideal of $A$. Let $M$ be a maximal Cohen-Macaulay A-module. Then the following condition are equivalent:

(1) $M$ is an Ulrich $A$-module with respect to $I$, and $I$ is a good ideal;

(2) $M$ is a weakly special Cohen-Macaulay A-module with respect to $I$.

Proof of Theorem 3.2. We may assume that $M / I M$ is $A / I$-free. Thus,

$$
\ell_{A}(M / I M)=\mu_{A}(M) \cdot \ell_{A}(A / I)
$$

$(1) \Longrightarrow(2)$ : By assumption we have

$$
\ell_{A}(M / I M)=e_{I}^{0}(M)=e_{I}^{0}(A) \cdot \operatorname{rank}_{A} M=2 \cdot \ell_{A}(A / I) \cdot \operatorname{rank}_{A} M,
$$

where the second equality follows from the associativity formula of multiplicities (e.g. [Ma, Theorem 14.8]). It follows from the above two equalities that $\mu_{A}(M)=2 \cdot \operatorname{rank}_{A} M$. Thus, $M$ is a weakly special Cohen-Macaulay $A$-module with respect to $I$.

$(2) \Longrightarrow(1)$ : Since $M$ is a maximal Cohen-Macaulay $A$-module, we have

$$
\ell_{A}(M / I M) \leqslant e_{I}^{0}(M) .
$$

On the other hand, by the observation and the equality described above, we get

$$
\begin{aligned}
\ell_{A}(M / I M) & =\mu_{A}(M) \cdot \ell_{A}(A / I)=2 \cdot \operatorname{rank}_{A} M \cdot \ell_{A}(A / I) \\
& \geqslant e_{I}^{0}(A) \cdot \operatorname{rank}_{A} M=e_{I}^{0}(M) .
\end{aligned}
$$

Therefore, $\ell_{A}(M / I M)=e_{I}^{0}(M)$ and $e_{I}^{0}(A)=2 \cdot \ell_{A}(A / I)$. That is, $M$ is an Ulrich $A$-module with respect to $I$ and $I$ is a good ideal. 
Corollary 3.3. Suppose that $A$ is a Gorenstein local domain. If I is an Ulrich ideal, then it is a weakly special ideal.

Proof. If $I$ is an Ulrich ideal, then it is a good ideal by Proposition 2.2, and $M=\operatorname{Syz}_{A}^{\operatorname{dim} A}(A / I)$ is an Ulrich $A$-module with respect to $I$ by Theorem 2.5. By Theorem 3.2, $M$ is a weakly special Cohen-Macaulay $A$ module with respect to $I$. Hence, $I$ is a weakly special ideal, as required.

Proposition 3.4. Suppose that $A$ is a hypersurface local domain. Then $I \subset A$ is an Ulrich ideal if and only if it is a weakly special ideal.

Proof. It suffices to prove the "if" part. Now suppose that $I$ is a weakly special ideal. Take a weakly special Cohen-Macaulay $A$-module $M$ with respect to $I$. By Theorem 3.2, $M$ is an Ulrich $A$-module with respect to $I$. Since $A$ is a hypersurface and $M$ is a maximal Cohen-Macaulay $A$-module without free summands, we have a minimal free presentation $A^{\mu} \rightarrow A^{\mu} \rightarrow$ $M \rightarrow 0$, which induces an exact sequence

$$
(A / Q)^{\mu} \rightarrow(A / Q)^{\mu} \stackrel{f}{\rightarrow} M / Q M \rightarrow 0 .
$$

As $M / Q M=M / I M$ is $A / I$-free, we have $M / Q M \cong(A / I)^{\mu}$. It is easy to observe that the kernel of $f$ is isomorphic to $(I / Q)^{\mu}$. Hence, there is a surjection $(A / Q)^{\mu} \rightarrow(I / Q)^{\mu}$, which shows $\mu_{A}(I / Q) \leqslant 1$. Thus, $\mu_{A}(I)=d+1$, and hence Proposition 2.2 implies that $I$ is an Ulrich ideal.

The following corollary gives a partial answer to Problem 1.3.

Corollary 3.5. Suppose that $A$ is a hypersurface local domain, and $I \subset A$ is a good ideal. If there exists an Ulrich $A$-module with respect to $I$, then $I$ is an Ulrich ideal.

Proof. The assertion follows from Theorem 3.2 and Proposition 3.4.

Question 3.6. Let $A$ be a Gorenstein local domain, and let $I \subset A$ be a stable ideal. Suppose that there exists an Ulrich $A$-module $M$ with respect to $I$. Is then $I$ an Ulrich ideal (especially, a good ideal)?

The next example shows that we cannot remove the assumption that $I$ is stable in Question 3.6.

ExAmPle 3.7. Let $k$ be a field, and let $e \geqslant 3$ be an integer. Set $A=$ $k\left[\left[t^{e}, t^{e+1}\right]\right]$ and $M=\left(t^{e}, t^{e+1}\right)^{e-1}$. Then $A$ is a hypersurface local domain and $M$ is an Ulrich $A$-module with respect to $\mathfrak{m}=\left(t^{e}, t^{e+1}\right)$, the maximal ideal of $A$. However, $\mathfrak{m}$ is not stable. 
The next example shows that we cannot relax the assumption that $A$ is a local domain in Question 3.6.

EXAMPLE 3.8. Let $k$ be a field, and let $a, e$ be integers with $2 a>e>$ $a \geqslant 2$. Set $A=k[[t]] /\left(t^{e}\right)$, and $I=\left(t^{a}\right)$. Then $I^{2}=0$ but $I \neq 0: I=\left(t^{e-a}\right)$. Hence, $I$ is stable but not good. Then $t^{e-a} A \cong A / I$ is an Ulrich $A$-module with respect to $I$.

\section{$\S 4$. Special Cohen-Macaulay modules over two-dimensional rational singularities}

Throughout this section, let $(A, \mathfrak{m})$ be a two-dimensional complete normal local domain with an algebraically closed residue field $k$ of characteristic zero. Let $\varphi: X \rightarrow \operatorname{Spec} A$ be the minimal resolution of singularities, with $E=\varphi^{-1}(\mathfrak{m})$ the exceptional divisor. Let $E=\bigcup_{j=1}^{r} E_{j}$ be the decomposition into irreducible components of $E$. Let $I \subset A$ be an $\mathfrak{m}$-primary ideal, and let $Q$ be a minimal reduction of $I$. For every maximal Cohen-Macaulay $A$-module $M$, we put $\widetilde{M}=\varphi^{*}(M) /$ torsion.

First, we recall the notion of special Cohen-Macaulay modules.

Theorem-Definition. [Special McKay correspondence due to Wunram] Assume that $A$ is a rational singularity, and let $\varphi: X \rightarrow \operatorname{Spec} A$ be as above. For every $i$, there exists a unique indecomposable maximal CohenMacaulay $A$-module $M_{i}$ (up to isomorphism) with $H^{1}\left(\widetilde{M}_{i}^{\vee}\right)=0$, so that

$$
c_{1}\left(\widetilde{M}_{i}\right) E_{j}=\delta_{i j}
$$

for every $1 \leqslant i, j \leqslant r$ and $\operatorname{rank}_{A} M_{i}=n_{i}$, where $c_{1}(\widetilde{M})$ denotes the first Chern class of $\widetilde{M}$ and $Z_{0}=\sum_{j=1}^{r} n_{j} E_{j}$ denotes the fundamental cycle on $X$.

Based upon this theorem, we define a (nontrivial) special CohenMacaulay $A$-module, which has been defined in more general settings.

Definition 4.1. (Special CM module) Suppose that $A$ is a twodimensional rational singularity. Let $M$ be a maximal Cohen-Macaulay $A$-module. Then $M$ is called a special Cohen-Macaulay A-module if $M$ is isomorphic to a finite direct sum of $M_{1}, \ldots, M_{r}$.

REMARK 4.2. Let $K_{A}$ denote the canonical module of $A$. A maximal Cohen-Macaulay $A$-module $M$ is said to be a special Cohen-Macaulay $A$ module if $M \otimes_{A} K_{A}$ /torsion is Cohen-Macaulay. This condition is equivalent to $\operatorname{Ext}_{A}^{1}(M, A)=0$ (see [IW]). In particular, any free $A$-module or any 
maximal Cohen-Macaulay module over a Gorenstein local domain $A$ is a special Cohen-Macaulay $A$-module in this sense. However, in this paper, we use the notion of special Cohen-Macaulay modules for two-dimensional rational singularities only.

Iyama and Wemyss [IW] proved the following characterization of special Cohen-Macaulay modules.

Proposition 4.3. (Cf. [IW, Theorem 3.6].) Suppose that $A$ is a twodimensional rational singularity. Let $M$ be a maximal Cohen-Macaulay $A$ module without free summands. Then $M$ is a special Cohen-Macaulay Amodule if and only if $\operatorname{Syz}_{A}^{1}(M) \cong M^{*}$, where $M^{*}=\operatorname{Hom}_{A}(M, A)$.

REMARK 4.4. Suppose that $A$ is a Gorenstein rational singularity, that is, $A$ is a rational double point. Then any maximal Cohen-Macaulay $A$ module is a finite direct sum of free modules and special Cohen-Macaulay $A$-modules.

As in the case of Ulrich modules, we define a special CM module with respect to an Ulrich ideal $I$.

Definition 4.5. (Special CM module with respect to $I$ ) Suppose that $A$ is a two-dimensional rational singularity with unique maximal ideal $\mathfrak{m}$, and let $I \subset A$ be an m-primary ideal. A finitely generated $A$-module $M$ is called a special Cohen-Macaulay A-module with respect to $I$ if the following conditions are satisfied:

(1) $M$ is a special Cohen-Macaulay $A$-module, that is, $\operatorname{Syz}_{A}^{1}(M) \cong M^{*}$;

(2) $M / I M$ is $A / I$-free.

Any special Cohen-Macaulay $A$-module is a weakly special CohenMacaulay $A$-module in the sense of 2.1 , but we believe that the converse is not true in general.

Lemma 4.6. Suppose that $A$ is a two-dimensional rational singularity. Let $M$ be a maximal Cohen-Macaulay A-module. Then:

(1) if $M$ is a special Cohen-Macaulay A-module with respect to $I$, then it is a weakly special Cohen-Macaulay A-module with respect to $I$;

(2) when $\operatorname{rank}_{A} M=1$, the converse of (1) holds true. 
Proof. (1) Suppose that $M$ is a special Cohen-Macaulay $A$-module. Then we have the following exact sequence:

$$
0 \rightarrow M^{*} \rightarrow A^{n} \rightarrow M \rightarrow 0
$$

where $n=\mu_{A}(M)$. This yields $\quad \mu_{A}(M)=\operatorname{rank}_{A} M+\operatorname{rank}_{A} M^{*}=$ $2 \cdot \operatorname{rank}_{A} M$.

(2) Take an ideal $J \subset A$ that is isomorphic to $M$. Then ht $J=1$ and $A / J$ is Cohen-Macaulay. It suffices to show that $\operatorname{Syz}_{A}^{1}(J) \cong J^{*}$.

As $\mu_{A}(J)=2 \cdot \operatorname{rank}_{A} J=2$, we can write $J=(x, y)$. Then

$$
\operatorname{Syz}_{A}^{1}(J) \cong\left\{\left[\begin{array}{c}
\alpha \\
\beta
\end{array}\right] \in A^{2} \mid \alpha x+\beta y=0\right\} \cong(x): y \cong J^{*} .
$$

Hence, $M \cong J$ is a special Cohen-Macaulay $A$-module with respect to $I$.

[Wu1, Example 1] is a weakly special Cohen-Macaulay $R$-module of rank 2 which is not special. After submitting the previous version of this paper, Yusuke Nakajima and the anonymous referee showed us such a concrete example [Na, Example 3.6, Example A.5]. See the example below.

REMARK 4.7. Let $k$ be an algebraically closed field of characteristic 0 , and let $G$ be the group

$$
G=\left\langle\left(\begin{array}{cc}
i & 0 \\
0 & -i
\end{array}\right),\left(\begin{array}{cc}
0 & i \\
i & 0
\end{array}\right),\left(\begin{array}{cc}
\xi_{6} & 0 \\
0 & \xi_{6}
\end{array}\right)\right\rangle=\mathbb{D}_{5,2} \subset \mathrm{GL}(2, k),
$$

where $i$ (resp. $\xi_{6}$ ) is a primitive fourth (resp. sixth) root of unity (see [Ri]).

Let $S=k[[s, t]]$ be the formal power series ring over $k$, and let $A=S^{G}$ be the invariant subring of $S$. Then the AR quiver of $A$ (see, e.g., [Y]) is the following (see, e.g., [IW, Example 4.6] and [Na, Example 3.6]).

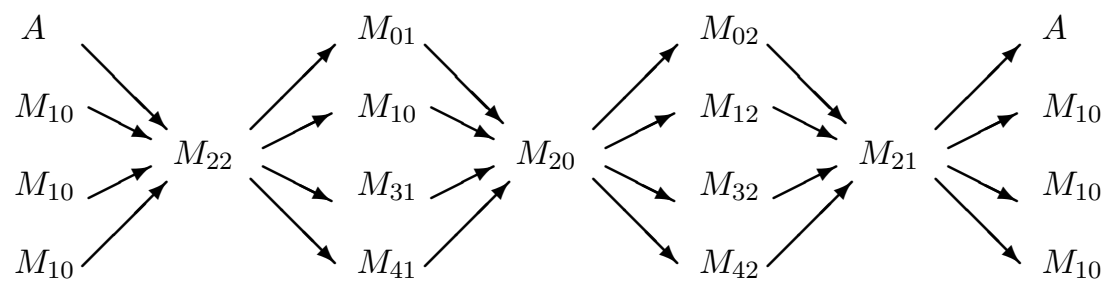

Then all indecomposable special Cohen-Macaulay modules of $A$ are $M_{02}$, $M_{11}, M_{31}$ and $M_{41}$ (see [IW, Example 4.6]). In particular, $M_{2,2}$ is an indecomposable maximal Cohen-Macaulay $A$-module which is not special. 
On the other hand, $\mu_{A}\left(M_{22}\right)=4$ and $\operatorname{rank}_{A} M_{22}=2$ by [Na, Example A.5]. Hence, $M_{22}$ is weakly special but not special (with respect to $\mathfrak{m}$ ).

Next, we introduce the notion of special ideals.

Definition 4.8. (Special ideal) An m-primary ideal $I \subset A$ is called a special ideal if it is a good ideal (cf. Definition 2.7) and there exists a special Cohen-Macaulay $A$-module $M$ (equivalently, $M_{j}$ for some $j$ ) with respect to $I$. When this is the case, such a cycle $Z$ is called a special cycle.

In the rest of this section, we give a characterization of special ideals in terms of cycles. Before doing that, we need the following lemma, which also plays an important role in Section 6 .

Let $Z=\sum_{i=1}^{r} a_{i} E_{i}$ and $W=\sum_{i=1}^{r} b_{i} E_{i}$ be anti-nef cycles on $X$. Put $\inf (Z, W)=\sum_{i=1}^{r} \inf \left(a_{i}, b_{i}\right) E_{i}$, then one can easily see that $\inf (Z, W)$ is also an anti-nef cycle on $X$.

Lemma 4.9. Assume that $Z \neq Z_{0}$ is an anti-nef cycle on $X$. Then we can find the following anti-nef cycles $Z_{1}, \ldots, Z_{s}$ and positive cycles $Y_{1}, \ldots, Y_{s}$ so that $0<Y_{s} \leqslant Y_{s-1} \leqslant \cdots \leqslant Y_{1} \leqslant Z_{0}$ :

$$
\left\{\begin{aligned}
Z=Z_{s} & =Z_{s-1}+Y_{s} \\
Z_{s-1} & =Z_{s-2}+Y_{s-1} \\
& \vdots \\
Z_{2} & =Z_{1}+Y_{2} \\
Z_{1} & =Z_{0}+Y_{1}
\end{aligned}\right.
$$

where $Z_{0}$ denotes the fundamental cycle on $X$.

Proof. We can take an integer $s \geqslant 1$ such that $Z \nless s Z_{0}$ and $Z \leqslant(s+$ 1) $Z_{0}$. Put $Z_{k}=\inf \left(Z,(k+1) Z_{0}\right)$ for every $k=1, \ldots, s$. Then $Z_{1}, \ldots, Z_{s}$ are anti-nef cycles. In particular, $Z_{0} \leqslant Z_{1} \leqslant Z_{2} \leqslant \cdots \leqslant Z_{s}=Z$. Moreover, if we put $Y_{k}=Z_{k}-Z_{k-1}$ for every $k=1, \ldots, s$, then we can obtain the required sequence.

Under the notation as in Lemma 4.9, we put $I_{k}=I_{Z_{k}}=H^{0}\left(X, \mathcal{O}_{X}\left(-Z_{k}\right)\right)$ for every $k=0,1, \ldots, s$. Then each $I_{k}$ is a good ideal and

$$
I=I_{s} \subset I_{s-1} \subset \cdots \subset I_{1} \subset I_{0}=\mathfrak{m} .
$$

The following theorem is the main theorem in this section, which gives a criterion for $I=I_{Z}$ to be a special ideal in terms of cycles. 
THEOREM 4.10. Let $Z=\sum_{j=1}^{r} a_{j} E_{j} \neq Z_{0}$ be an anti-nef cycle on the minimal resolution $X \rightarrow \operatorname{Spec} A$, and put $I=I_{Z}$. Let $Z_{0}=\sum_{j=1}^{r} n_{j} E_{j}$ denote the fundamental cycle on $X$. Suppose that $1 \leqslant i \leqslant r$. Then the following conditions are equivalent:

(1) $M_{i}$ is a special Cohen-Macaulay A-module with respect to I;

(2) $a_{i}=n_{i} \cdot \ell_{A}(A / I)$;

(3) there exist positive cycles $0<Y_{s} \leqslant \cdots \leqslant Y_{1} \leqslant Z_{0}$ and anti-nef cycles $Z_{1}, \ldots, Z_{s}$ so that $Z_{k}=Z_{k-1}+Y_{k}$ for each $k=1, \ldots, s$ and

$$
\begin{aligned}
& Z_{k-1} \cdot Y_{k}=0, \quad p_{a}\left(Y_{k}\right)=0 \text { and } \operatorname{coeff}_{E_{i}} Y_{k}=n_{i} \\
& \quad \text { for every } k=1,2, \ldots, s,
\end{aligned}
$$

where coeff $E_{i} W$ stands for the coefficient of $E_{i}$ in a cycle $W$.

When this is the case, $\ell_{A}(A / I)=s+1$ and every $I_{k}:=I_{Z_{k}}$ is a special ideal. Moreover, for every $k=1,2, \ldots, s$, we obtain that $\operatorname{Supp}\left(Y_{k}\right)$ is given as one of the connected components of $\cup\left\{E_{j} \subset \operatorname{Supp}\left(Y_{k-1}\right) \mid E_{j} Z_{k-1}=0\right\}$, and $Y_{k}$ is the fundamental cycle on $\operatorname{Supp}\left(Y_{k}\right)$.

REMARK 4.11. $Y+Z$ is not necessarily anti-nef even if $Y$ is the fundamental cycle on $X$ that satisfies $p_{a}(Y)=0$ and $Y Z=0$.

Let us begin the proof of Theorem 4.10. The following formula is one of the main tools in this paper.

LEMmA 4.12. (Kato's Riemann-Roch formula; [Ka, WY]) Let $Z$ be an anti-nef cycle on the minimal resolution of singularities $X$, and put $I_{Z}=$ $H^{0}\left(X, \mathcal{O}_{X}(-Z)\right)$. Then for any maximal Cohen-Macaulay A-module $M$, we have

$$
\ell_{A}\left(M / I_{Z} M\right)=\operatorname{rank}_{A} M \cdot \ell_{A}\left(A / I_{Z}\right)+c_{1}(\widetilde{M}) Z
$$

In particular,

$$
\ell_{A}\left(A / I_{Z}\right)=-\frac{Z^{2}+K_{X} Z}{2}=1-p_{a}(Z) .
$$

The next lemma easily follows from Lemma 4.12.

LEMmA 4.13. Under the notation as in Theorem 4.10, we have

$$
\ell_{A}\left(A / I_{k}\right)=\ell_{A}\left(A / I_{k-1}\right)-Y_{k} Z_{k-1}+1-p_{a}\left(Y_{k}\right)
$$


Proof. By Lemma 4.12, we have

$$
\begin{aligned}
\ell_{A}\left(A / I_{k}\right) & =1-p_{a}\left(Z_{k}\right)=1-p_{a}\left(Z_{k-1}+Y_{k}\right) \\
& =1-p_{a}\left(Z_{k-1}\right)-p_{a}\left(Y_{k}\right)-Y_{k} Z_{k-1}+1 \\
& =\ell_{A}\left(A / I_{k-1}\right)-Y_{k} Z_{k-1}+1-p_{a}\left(Y_{k}\right),
\end{aligned}
$$

as required.

The following lemma is a key lemma in the proof of the theorem.

LEMMA 4.14. Under the notation as in Theorem 4.10, we have

(1) $a_{i} \leqslant n_{i} \cdot \ell_{A}(A / I)$;

(2) equality holds in (1) if and only if $M_{i}$ is a special Cohen-Macaulay A-module with respect to $I$.

Proof. By Kato's Riemann-Roch formula, we have

$$
\ell_{A}\left(M_{i} / I M_{i}\right)=\operatorname{rank}_{A} M_{i} \cdot \ell_{A}(A / I)+c_{1}\left(\widetilde{M}_{i}\right) \cdot Z=n_{i} \cdot \ell_{A}(A / I)+a_{i}
$$

On the other hand, $\mu_{A}\left(M_{i}\right)=2 n_{i}$ because $M_{i}$ is a special Cohen-Macaulay $A$-module (with respect to $\mathfrak{m}$ ). Hence,

$$
\ell_{A}\left(M_{i} / I M_{i}\right) \leqslant \mu_{A}\left(M_{i}\right) \cdot \ell_{A}(A / I)=2 n_{i} \cdot \ell_{A}(A / I) .
$$

Therefore, $a_{i} \leqslant n_{i} \cdot \ell_{A}(A / I)$ and equality holds true if and only if $M_{i} / I M_{i}$ is $A / I$-free, which means that $M_{i}$ is a special Cohen-Macaulay $A$-module with respect to $I$.

Proof of Theorem 4.10. (1) $\Longleftrightarrow$ (2) follows from Lemma 4.14.

$(2) \Longrightarrow(3)$ : Choose anti-nef cycles $Z_{1}, \ldots, Z_{s}$ and positive cycles $Y_{1}, \ldots, Y_{s}$ that satisfy the condition of Lemma 4.9 . For these $Y_{i}$ s and $Z_{i}$ s, we use induction on $s$. By Lemma 4.13, we have

$$
\begin{aligned}
n_{i} \cdot \ell_{A}(A / I)-\operatorname{coeff}_{E_{i}} Z \\
=n_{i}\left\{\ell_{A}\left(A / I_{s-1}\right)-Y_{s} Z_{s-1}+1-p_{a}\left(Y_{s}\right)\right\}-\operatorname{coeff}_{E_{i}} Z_{s-1}-\operatorname{coeff}_{E_{i}} Y_{s} \\
=\left\{n_{i} \cdot \ell_{A}\left(A / I_{s-1}\right)-\operatorname{coeff}_{E_{i}} Z_{s-1}\right\}+n_{i}\left(-Y_{s} Z_{s-1}\right)+n_{i}\left(-p_{a}\left(Y_{s}\right)\right) \\
\quad+\left\{n_{i}-\operatorname{coeff}_{E_{i}} Y_{s}\right\} .
\end{aligned}
$$

By the induction hypothesis, $n_{i} \cdot \ell_{A}\left(A / I_{s-1}\right)-$ coeff $_{E_{i}} Z_{s-1} \geqslant 0$. Since $Z_{s-1}$ is anti-nef, $-Y_{s} Z_{s-1} \geqslant 0$. As $A$ is rational, $-p_{a}\left(Y_{s}\right) \geqslant 0$. By the choice of $Y_{s}$, 
$n_{i}-\operatorname{coeff}_{E_{i}} Y_{s} \geqslant 0$. Hence, we obtain the required inequality, and equality holds if and only if

$$
\begin{aligned}
& n_{i} \cdot \ell_{A}\left(A / I_{s-1}\right)=\operatorname{coeff}_{E_{i}} Z_{s-1}, \\
& Y_{s} Z_{s-1}=p_{a}\left(Y_{s}\right)=0 \text { and } n_{i}=\operatorname{coeff}_{E_{i}} Y_{s} .
\end{aligned}
$$

Therefore, the assertion follows from the induction hypothesis. We can prove the converse $(3) \Longrightarrow(2)$ similarly.

Now suppose that one of (1), (2), (3) holds. The induction hypothesis implies that $I_{s-1}$ is a special ideal with $\ell\left(A / I_{s-1}\right)=s, \operatorname{Supp}\left(Y_{k}\right)$ is connected, $\operatorname{Supp}\left(Y_{k}\right) \subset \cup\left\{E_{j} \mid E_{j} Z_{k-1}=0\right\}$ and $Y_{k}$ is the fundamental cycle on $\operatorname{Supp}\left(Y_{k}\right)$ for every $k=1, \ldots, s-1$. Then it follows from Lemma 4.13 that $\ell\left(A / I_{s}\right)=\ell\left(A / I_{s-1}\right)+1=s+1$. As $Y_{s} Z_{s-1}=0$, it follows that $\operatorname{Supp}\left(Y_{s}\right) \subset$ $\cup\left\{E_{j} \mid E_{j} Z_{s-1}=0\right\}$. Moreover, since $p_{a}\left(Y_{s}\right)=0, Y_{s}$ must be connected.

Let us show that $Y_{s}$ is the fundamental cycle on $\operatorname{Supp}\left(Y_{s}\right)$. For each $E_{j} \subset \operatorname{Supp}\left(Y_{s}\right), Y_{s} E_{j}=Y_{s} E_{j}+Z_{s-1} E_{j}=Z_{s} E_{j} \leqslant 0$. Hence, $Y_{s}$ is anti-nef on $\operatorname{Supp}\left(Y_{s}\right)$. If $Y_{s}$ is not the fundamental cycle on $\operatorname{Supp}\left(Y_{s}\right)$, then there exist an $E_{j} \subset \operatorname{Supp}\left(Y_{s}\right)$ and an anti-nef cycle $Y_{s}^{\prime}$ on $\operatorname{Supp}\left(Y_{s}\right)$ so that $Y_{s}=Y_{s}^{\prime}+E_{j}$. Then

$$
0=p_{a}\left(Y_{s}\right)=p_{a}\left(Y_{s}^{\prime}\right)+p_{a}\left(E_{j}\right)+Y_{s}^{\prime} E_{j}-1 \leqslant p_{a}\left(Y_{s}^{\prime}\right)-1 \leqslant-1 .
$$

This is a contradiction.

\section{$\S 5$. Ulrich ideals and modules over rational double points}

The goal of this section is to classify Ulrich ideals of any two-dimensional Gorenstein rational singularity (rational double point) $A$, and to determine all of the Ulrich $A$-modules with respect to those ideals.

First, we recall the definition of rational double points.

Definition 5.1. (Rational double point) Let $A$ be a two-dimensional complete Noetherian local ring with unique maximal ideal $\mathfrak{m}$ containing an algebraically closed field $k$. Then $A$ is said to be a rational double point if it is isomorphic to the hypersurface $k[[x, y, z]] /(f)$, where $f$ is one of the following polynomials:

$$
\begin{array}{lll}
\left(A_{n}\right) & z^{2}+x^{2}+y^{n+1} & (n \geqslant 1) \\
\left(D_{n}\right) & z^{2}+x^{2} y+y^{n-1} \quad(n \geqslant 4) \\
\left(E_{6}\right) & z^{2}+x^{3}+y^{4} \\
\left(E_{7}\right) & z^{2}+x^{3}+x y^{3} \\
\left(E_{8}\right) & z^{2}+x^{3}+y^{5}
\end{array}
$$


By flat base change, it is known that $A$ is a two-dimensional local Gorenstein rational singularity (of characteristic 0 ) if and only if the $\mathfrak{m}$-adic completion $\widehat{A}$ is a rational double point in the above sense [Vi, Remarks p.1].

The following theorem is the first main result in this section. In the latter half of this section, we give the complete classification of Ulrich ideals and modules as an application of the theorem.

TheOREm 5.2. (See also Theorem 3.2.) Assume that $A$ is a rational double point of dimension 2 , and let $I \subset A$ be a nonparameter $\mathfrak{m}$-primary ideal. Suppose that $M$ is a maximal Cohen-Macaulay A-module. Then the following conditions are equivalent:

(1) $M$ is an Ulrich $A$-module with respect to $I$;

(2) $M$ is a special Cohen-Macaulay A-module with respect to I;

(3) $M$ is a weakly special Cohen-Macaulay A-module with respect to I;

(4) $M / I M$ is $A / I$-free and $M$ has no free summands.

When this is the case, $I$ is an Ulrich ideal and $M^{*} \cong \operatorname{Syz}_{A}^{1}(M)$ is also an Ulrich A-module with respect to $I$.

In what follows, we prove Theorem 5.2. We need several lemmas. Although the following lemma can be proved in a similar method to the proof of Theorem 3.2, we do not need to assume that $I$ is stable here.

Lemma 5.3. Assume that $A$ is a rational double point of dimension 2, and let $I \subset A$ be an $\mathfrak{m}$-primary ideal. Then $e_{I}^{0}(A) \leqslant 2 \cdot \ell_{A}(A / I)$ holds true and equality holds if and only if $I$ is a good ideal.

Proof. The lemma is well known, but we give a proof here for the convenience of the reader. Let $\bar{I}$ denote the integral closure of $I$. Take a minimal reduction $Q$ of $I$. Then since $Q$ is also a minimal reduction of $\bar{I}$ and $\bar{I}^{2}=Q \bar{I}$, we have

$$
I \subset \bar{I} \subset Q: \bar{I} \subset Q: I
$$

The Matlis duality theorem implies that

$$
\begin{aligned}
e_{I}^{0}(A) & =\ell_{A}(A / Q)=\ell_{A}(A / I)+\ell_{A}(I / Q) \leqslant \ell_{A}(A / I)+\ell_{A}(Q: I / Q) \\
& =2 \cdot \ell_{A}(A / I),
\end{aligned}
$$

and equality holds if and only if $I=Q: I$, that is, $I$ is a good ideal. 
Almost all of the maximal Cohen-Macaulay $A$-modules over a hypersurface of multiplicity 2 can be regarded as Ulrich modules in the classical sense.

Lemma 5.4. (Cf. [HKuh, Corollary 1.4].) Let $A$ be a hypersurface local domain of $e_{\mathfrak{m}}^{0}(A)=2$. Then every maximal Cohen-Macaulay A-module without free summands satisfies $\mu_{A}(M)=e_{\mathfrak{m}}^{0}(M)=2 \cdot \operatorname{rank}_{A} M$, that is, $M$ is an Ulrich A-module with respect to $\mathfrak{m}$.

Any two-dimensional rational double point $A$ can be regarded as an invariant subring $B^{G}$, where $B=k[[s, t]]$ is a formal power series ring over $k$, and $G$ is a finite subgroup of $\operatorname{SL}(2, k)$. Thus, we can apply the socalled McKay correspondence, which is a special case of "special McKay correspondence" (see Section 4).

Lemma 5.5. (McKay correspondence) Let $A=B^{G}$ as above. Then we have the following.

(1) The ring $A$ is of finite CM-representation type. Let $\left\{M_{i}\right\}_{i=0}^{r}$ be the set of isomorphism classes of indecomposable maximal Cohen-Macaulay $A$ modules, where $M_{0}=A$. Then $B \cong \bigoplus_{i=0}^{r} M_{i}^{\oplus n_{i}}$, where $n_{i}=\operatorname{rank}_{A} M_{i}$.

(2) The fundamental cycle is given by $Z_{0}=\sum_{j=1}^{r} n_{j} E_{j}$, so that if we choose indices suitably, then $c_{1}\left(\widetilde{M}_{i}\right) E_{j}=\delta_{i j}$ for $1 \leqslant i, j \leqslant r$, where $c_{1}(*)$ denotes the Chern class, and $\widetilde{M}_{i}=\varphi^{*}\left(M_{i}\right) /$ torsion. In particular, $M_{i}$ is a special Cohen-Macaulay A-module (with respect to $\mathfrak{m}$ ) for every $i=1,2, \ldots, r$.

We are now ready to prove Theorem 5.2.

Proof of Theorem 5.2. (1) $\Longrightarrow(2)$ : Since $M$ is an Ulrich $A$-module with respect to $I$, it has no free summands because no free module is an Ulrich $A$-module with respect to $I$. Thus, $M$ is an Ulrich $A$-module with respect to $\mathfrak{m}$ by Lemma 5.4 , and it is also a special Cohen-Macaulay $A$-module with respect to $\mathfrak{m}$ by Lemma 5.5 . Hence, $M$ is a special Cohen-Macaulay $A$-module with respect to $I$ because $M / I M$ is $A / I$-free.

$(2) \Longrightarrow(3)$ : See Lemma 4.6.

$(3) \Longrightarrow(4)$ : Trivial.

$(4) \Longrightarrow(1)$ : By Lemma 5.4, $M$ is a weakly special Cohen-Macaulay $A$ module with respect to $I$. Note that $e_{I}^{0}(A) \leqslant 2 \cdot \ell_{A}(A / I)$ by Lemma 5.3 . By a similar argument to the proof of Theorem 3.2, we have

$$
\ell_{A}(M / I M)=e_{I}^{0}(M) \text { and } e_{I}^{0}(A)=2 \cdot \ell_{A}(A / I),
$$

whence $M$ is an Ulrich $A$-module with respect to $I$, and $I$ is a good ideal. 
Since $A$ is a hypersurface local domain, Proposition 3.4 implies that $I$ is an Ulrich ideal. In particular, $A / I$ is Gorenstein. Thus, application of Theorem 2.6 yields that $M^{*} \cong \operatorname{Syz}_{A}^{1}(M)$ is also an Ulrich $A$-module with respect to $I$.

The corollary below follows from Proposition 3.4 and Theorem 5.2.

Corollary 5.6. Assume that $A$ is a rational double point of dimension 2. Let I be an $\mathfrak{m}$-primary ideal. Then the following conditions are equivalent:

(1) I is an Ulrich ideal;

(2) I is a special ideal;

(3) I is a weakly special ideal;

(4) there exists an Ulrich A-module with respect to $I$.

In the rest of this section, we classify all Ulrich ideals and Ulrich modules over rational double points of dimension 2 using the results in the previous section.

Let $\left\{M_{i}\right\}_{i=0}^{r}$ be the set of indecomposable maximal Cohen-Macaulay $A$ modules, so that $M_{0}=A$ and $c_{1}\left(\widetilde{M}_{i}\right) E_{j}=\delta_{i j}$ for all $1 \leqslant i, j \leqslant r$.

Now suppose that $M$ is an Ulrich $A$-module with respect to $I$. Then $M$ is a finite direct sum of $M_{1}, \ldots, M_{r}$ :

$$
M \cong M_{1}^{\oplus k_{1}} \oplus \cdots \oplus M_{r}^{\oplus k_{r}},
$$

because $M$ has no free summands. Whenever $k_{i}>0, M_{i}$ must be an Ulrich $A$-module with respect to $I$. Hence, it suffices to characterize all $M_{i}$ that are Ulrich $A$-modules with respect to $I$. On the other hand, Theorem 5.2 implies that $I$ is an Ulrich ideal, whence $I$ is a special ideal. Thus, those ideals $I$ (or cycles $Z$ ) are determined by Theorem 4.10. Moreover, it is not difficult to determine all $M_{i}$ that are Ulrich $A$-modules with respect to $I_{Z}$ by Theorem 5.2 .

Let $I$ be a good ideal of $A$, and let $Z$ be an anti-nef cycle on the minimal resolution $X$ such that $I \mathcal{O}_{X}=\mathcal{O}_{X}(-Z)$ and $I=\mathrm{H}^{0}\left(X, \mathcal{O}_{X}(-Z)\right)$, that is, $I=I_{Z}$. Then we call $Z$ an Ulrich cycle if $I$ is an Ulrich ideal. Note that by Corollary 5.6, $Z$ is an Ulrich cycle if and only if it is a special cycle.

Now let us illustrate the main theorem by the following example. Let $Z=2 E_{1}+3 E_{2}+4 E_{3}+3 E_{4}+2 E_{5}+2 E_{6}$ be the cycle of the rational double point $A=k[[x, y, z]] /\left(x^{3}+y^{4}+z^{2}\right)$, and put $I=\mathrm{H}^{0}\left(X, \mathcal{O}_{X}(-Z)\right)$. Then 
since $Z$ is an anti-nef cycle on the minimal resolution $X \rightarrow \operatorname{Spec} A$ with support in $E=\bigcup_{i=1}^{6} E_{i}, Z$ can be described as follows.

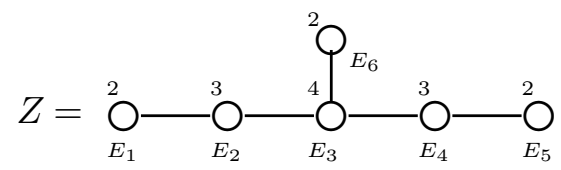

Furthermore, by Theorem 4.10(2), $M_{i}$ is an Ulrich $A$-module with respect to $I$ if and only if $i=1$ or 5 , because $Z_{0}=E_{1}+2 E_{2}+3 E_{3}+2 E_{4}+E_{5}+2 E_{6}$ and $\ell_{A}(A / I)=2$. In other words, any Ulrich $A$-module with respect to $I$ is given by $M \cong M_{1}^{\oplus a} \oplus M_{5}^{\oplus b}$ for some integers $a, b \geqslant 0$. We can describe this by the following picture.

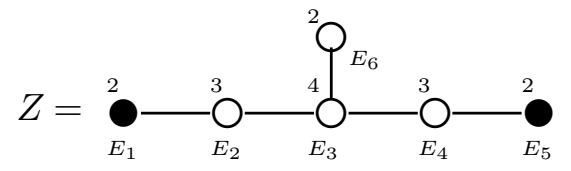

We are now ready to state the main theorem in this section.

THEOREM 5.7. Let $A$ be a two-dimensional rational double point. Let $\varphi$ : $X \rightarrow \operatorname{Spec} A$ be the minimal resolution of singularities with $E=\varphi^{-1}(\mathfrak{m})=$ $\bigcup_{i=1}^{r} E_{i}$, the exceptional divisor on $X$. Then all Ulrich cycles $Z_{k}$ of $A$ and all indecomposable Ulrich $A$-modules with respect to $I_{k}=\mathrm{H}^{0}\left(X, \mathcal{O}_{X}\left(-Z_{k}\right)\right)$ are given by the following.

- $\left(A_{n}\right) x^{2}+y^{n+1}+z^{2}$

When $n=2 m$, the complete list of all Ulrich cycles is given by the following:

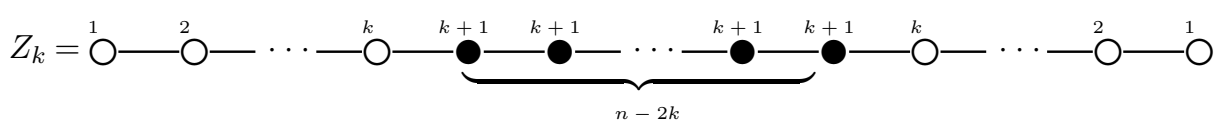

for $k=0,1, \ldots, m-1\left(=\frac{n}{2}-1\right)$. Then $\ell_{A}\left(A / I_{k}\right)=k+1$ for each $k=$ $0,1, \ldots, m-1$.

When $n=2 m+1$, the complete list of all Ulrich cycles is given by the following:

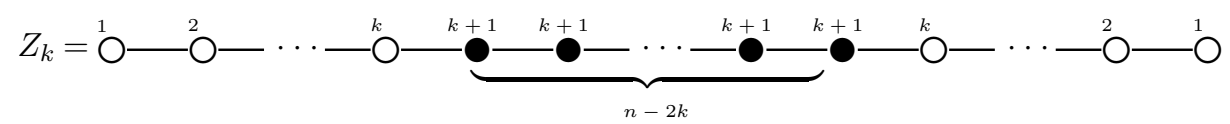

for $k=0,1, \ldots, m\left(=\frac{n-1}{2}\right)$. Then $\quad \ell_{A}\left(A / I_{k}\right)=k+1 \quad$ for $\quad$ each $\quad k=$ $0,1, \ldots, m$. 
- $\left(D_{n}\right) x^{2} y+y^{n-1}+z^{2}(n \geqslant 4)$

When $n=2 m$, the complete list of all Ulrich cycles is given by the following:

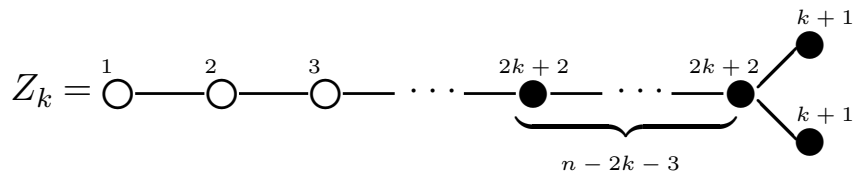

for $k=0,1, \ldots, m-2\left(=\frac{n-4}{2}\right)$.
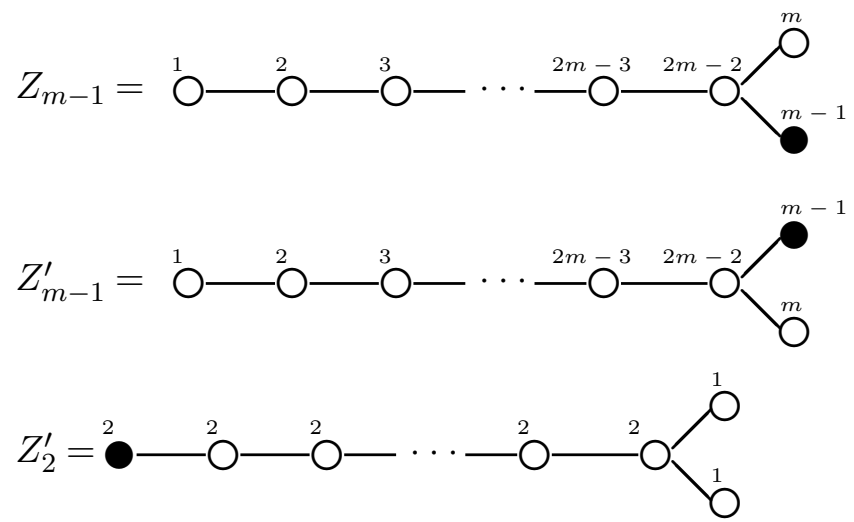

Then $\ell_{A}\left(A / I_{k}\right)=k+1 \quad$ for $\quad$ each $k=0,1, \ldots, m-2, \quad \ell_{A}\left(A / I_{m-1}\right)=$ $\ell_{A}\left(A / I_{m-1}^{\prime}\right)=m$ and $\ell_{A}\left(A / I_{2}^{\prime}\right)=2$, where $m=\frac{n}{2}$.

When $n=2 m+1$, the complete list of all Ulrich cycles is given by the following:

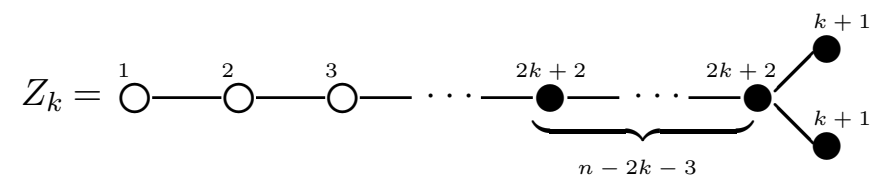

for $k=0,1, \ldots, m-2\left(=\frac{n-5}{2}\right)$.

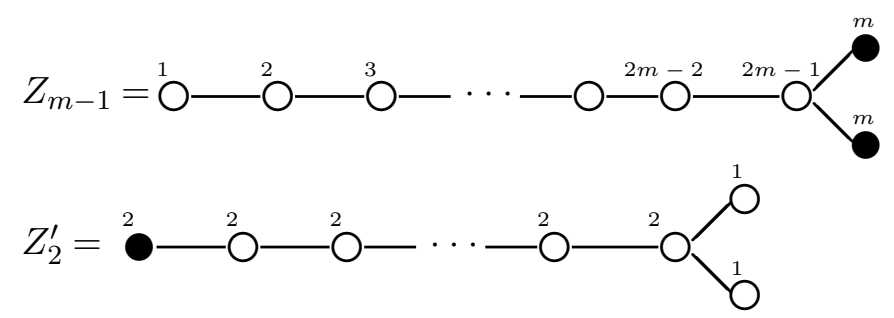

Then $\ell_{A}\left(A / I_{k}\right)=k+1$ for each $k=0,1, \ldots, m-1$, and $\ell_{A}\left(A / I_{2}^{\prime}\right)=2$. 
- $\left(E_{6}\right) x^{3}+y^{4}+z^{2}$

The Ulrich cycles of $A$ are the following $Z_{0}$ and $Z_{1}$ with $\ell_{A}\left(A / I_{k}\right)=k+1$ for each $k=0,1$ :

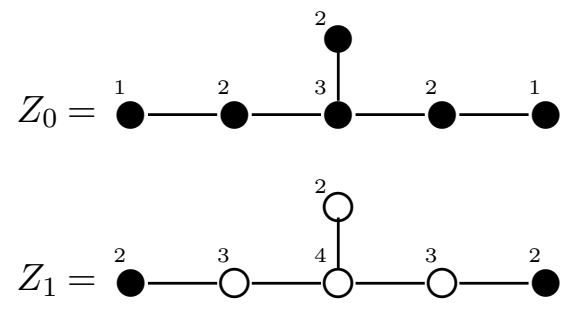

- $\left(E_{7}\right) x^{3}+x y^{3}+z^{2}$

The Ulrich cycles of $A$ are the following $Z_{0}, Z_{1}$ and $Z_{2}$ with $\ell_{A}\left(A / I_{k}\right)=$ $k+1$ for each $k=0,1,2$ :
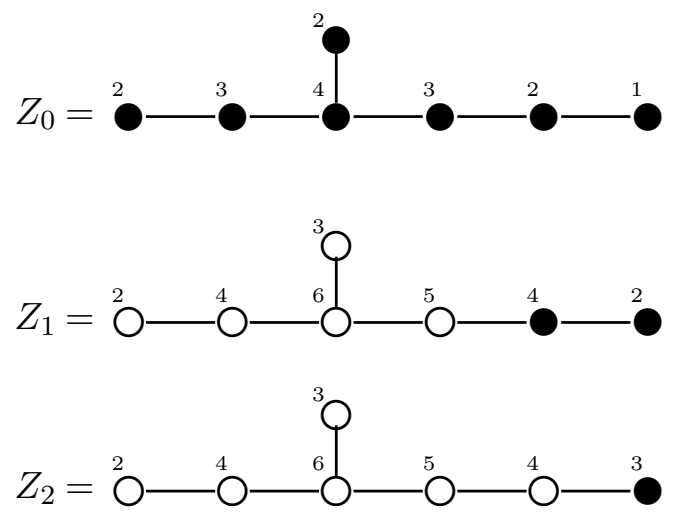

- $\left(E_{8}\right) x^{3}+y^{5}+z^{2}$

The Ulrich cycles of $A$ are the following $Z_{0}$ and $Z_{1}$ with $\ell_{A}\left(A / I_{k}\right)=k+1$ for each $k=0,1$ :

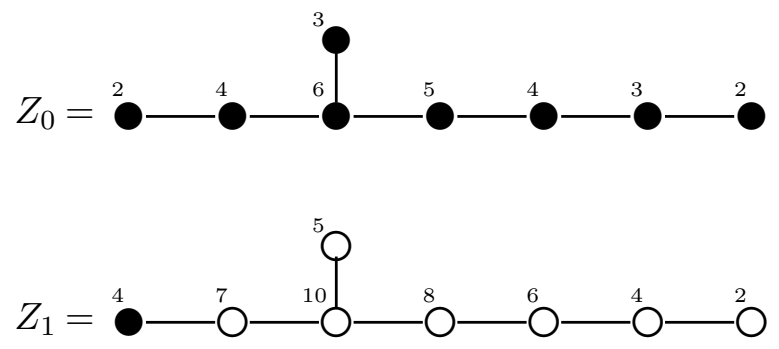


In our previous paper [GOTWY, Section 9], we gave a complete list of the nonparameter Ulrich ideals for one-dimensional simple singularities. We can also do this for two-dimensional simple singularities (rational double points).

Corollary 5.8. With the same notation as in Theorem 5.7, the set $\mathcal{X}_{A}$ is equal to the following.

$$
\begin{aligned}
&\left(A_{2 m}\right)\left\{(x, y, z),\left(x, y^{2}, z\right), \ldots,\left(x, y^{m}, z\right)\right\} . \\
&\left(A_{2 m+1}\right)\left\{(x, y, z),\left(x, y^{2}, z\right), \ldots,\left(x, y^{m+1}, z\right)\right\} . \\
&\left(D_{2 m}\right) \quad\left\{(x, y, z),\left(x, y^{2}, z\right), \ldots,\left(x, y^{m-1}, z\right),\right.\left.\left(x+\sqrt{-1} y^{m-1}, y^{m}, z\right),\left(x-\sqrt{-1} y^{m-1}, y^{m}, z\right),\left(x^{2}, y, z\right)\right\} . \\
&\left(D_{2 m+1}\right) \quad\left\{(x, y, z),\left(x, y^{2}, z\right), \ldots,\left(x, y^{m}, z\right),\left(x^{2}, y, z\right)\right\} . \\
&\left(E_{6}\right) \quad\left\{(x, y, z),\left(x, y^{2}, z\right)\right\} . \\
&\left(E_{7}\right) \quad\left\{(x, y, z),\left(x, y^{2}, z\right),\left(x, y^{3}, z\right)\right\} . \\
&\left(E_{8}\right) \quad\left\{(x, y, z),\left(x, y^{2}, z\right)\right\} .
\end{aligned}
$$

Proof. One can easily see that any ideal $I$ appearing in the corollary has the form $I=Q+(z)$, where $Q$ is a parameter ideal of $A$ and $I^{2}=Q I$, $\ell_{A}(A / Q)=2 \cdot \ell_{A}(A / I)$ and $\mu(I)=3$. Hence, those ideals $I$ are Ulrich.

On the other hand, Theorem 5.7 implies that $\sharp \mathcal{X}_{A}=m$ (resp. $m+1, m+$ $2, m+1,2,3,2)$ if $A$ is a rational double point of type $\left(A_{2 m}\right)\left(\operatorname{resp} .\left(A_{2 m+1}\right)\right.$, $\left.\left(D_{2 m}\right),\left(D_{2 m+1}\right),\left(E_{6}\right),\left(E_{7}\right),\left(E_{8}\right)\right)$. Hence, the set as above coincides with $\mathcal{X}_{A}$, respectively.

Proof of Theorem 5.7. We first consider the cases $\left(E_{6}\right),\left(E_{7}\right),\left(E_{8}\right)$.

The case $\left(E_{6}\right): f=x^{3}+y^{4}+z^{2}$. The fundamental cycle $Z_{0}$ on the minimal resolution is given by

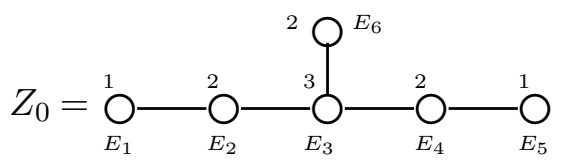

Now suppose that $Z_{0}+Y$ is a special cycle for some positive cycle $Y \leqslant Z_{0}$. Since $\cup\left\{E \mid E Z_{0}=0\right\}=\bigcup_{i=1}^{5} E_{i}$ is connected, we have $Y=Y_{1}:=\sum_{i=1}^{5} E_{i}$ in 
Theorem 4.10(3). Conversely, if we put

$$
Z_{1}=Z_{0}+Y_{1}=\bigcirc_{E_{1}}^{2}-\bigcirc_{E_{2}}^{3}-{ }_{E_{3}}^{Q^{E_{6}}}-\stackrel{\bigcirc_{E_{4}}^{3}}{\overbrace{E_{5}}^{2}}
$$

then $Z_{1}$ is anti-nef and $p\left(Y_{1}\right)=0$ because $Y_{1}$ can be regarded as the fundamental cycle on the dual graph (of the minimal resolution) of type $\left(A_{5}\right)$. Hence, $Z_{1}$ is a special cycle and $M$ is an Ulrich $A$-module with respect to $I_{Z_{1}}$ if and only if it is a finite direct sum of $M_{1}$ and $M_{5}$, because coeff $_{E_{i}} Y_{1}=n_{i}(=1) \Longleftrightarrow i=1,5$ (see Theorem 4.10).

Suppose that $Z_{2}=Z_{1}+Y$ is a special cycle for some positive cycle $Y \leqslant Y_{1}$. As $\cup\left\{E \subset \operatorname{Supp}\left(Y_{1}\right) \mid E Z_{1}=0\right\}=E_{2} \cup E_{3} \cup E_{4}$ is connected, we have that $Y=E_{2}+E_{3}+E_{4}$ as the fundamental cycle on $\operatorname{Supp}(Y)=E_{2} \cup$ $E_{3} \cup E_{4}$. However, $Z_{2}=Z_{1}+Y=2 E_{1}+4 E_{2}+5 E_{3}+4 E_{4}+2 E_{5}+2 E_{6}$ is not anti-nef because $Z_{2} E_{6}=1$. Therefore, the special cycles of $\left(E_{6}\right)$ are $Z_{0}$ and $Z_{1}$.

The case $\left(E_{7}\right): f=x^{3}+x y^{3}+z^{2}$. The fundamental cycle $Z_{0}$ on the minimal resolution is given by

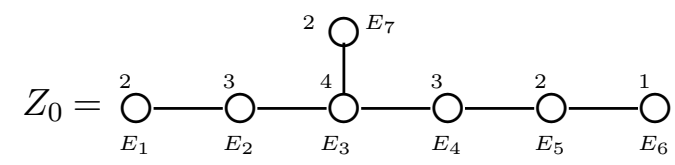

Since $\cup\left\{E \mid E Z_{0}=0\right\}=\bigcup_{i=2}^{7} E_{i}$ is isomorphic to the dual graph of $\left(D_{6}\right)$, if $Z_{1}=Z_{0}+Y$ is a special cycle for some positive cycle $Y \leqslant Z_{0}$, then we have

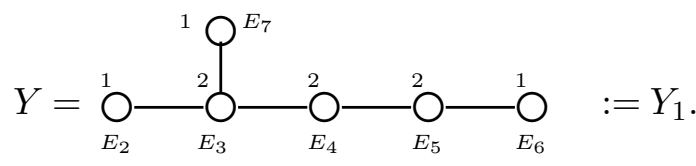

Conversely, one can easily see that the following $Z_{1}$ is a special cycle by Theorem 4.10.

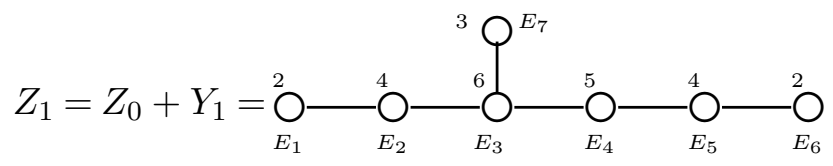


Note that $\cup\left\{E \subset \operatorname{Supp}\left(Y_{1}\right) \mid E Z_{1}=0\right\}$ admits two connected components:

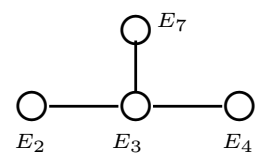

The fundamental cycles $Y_{2}$ of their components are $E_{2}+2 E_{3}+E_{4}+E_{5}$ and $E_{6}$, respectively. Note that $Z_{1}+E_{6}$ is anti-nef but $Z_{1}+\left(E_{2}+2 E_{3}+E_{4}+\right.$ $\left.E_{5}\right)$ is not anti-nef. Therefore, we are done.

The case $\left(E_{8}\right): x^{3}+y^{5}+z^{2}$. The fundamental cycle $Z_{0}$ on the minimal resolution is given by

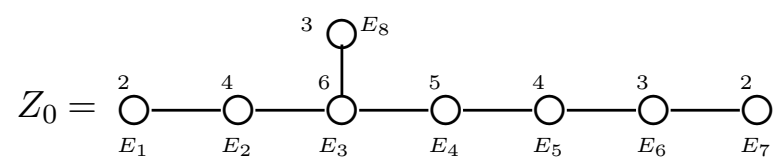

Suppose that $Z_{0}+Y$ is a special cycle for some positive cycle $Y \leqslant Z_{0}$. As $\cup\left\{E \mid E Z_{0}=0\right\}=\bigcup_{i \neq 7} E_{i}$ is connected and the corresponding graph is isomorphic to the dual graph of $\left(E_{7}\right)$, we have

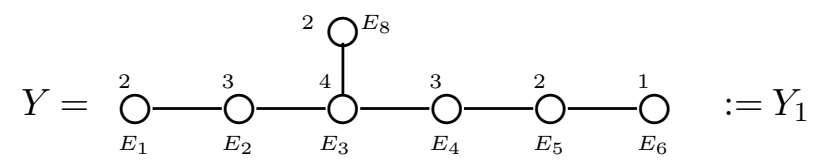

Conversely, if we put

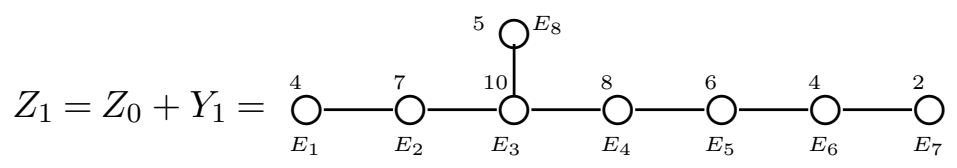

then $Z_{1}$ is a special cycle by Theorem 4.10 .

Now suppose that $Z_{1}+Y$ is a special cycle for some positive cycle $Y \leqslant Y_{1}$. Since $\cup\left\{E \subset \operatorname{Supp}\left(Y_{1}\right) \mid E Z_{1}=0\right\}=E_{2} \cup E_{3} \cup E_{4} \cup E_{5} \cup E_{6} \cup E_{8}$ is connected, we have $Y=E_{2}+2 E_{3}+2 E_{4}+2 E_{5}+E_{6}+E_{8}$. However, $Z_{1}+Y$ is not anti-nef.

We next consider the case $\left(A_{n}\right)$.

The case $\left(A_{2 m}\right): f=x^{2}+y^{2 m+1}+z^{2}$. The fundamental cycle $Z_{0}$ on the minimal resolution is given by

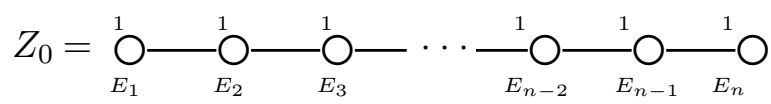

That is, $Z_{0}=\sum_{i=1}^{n} E_{i}$. 
Now suppose that $Z_{0}+Y$ is a special cycle for some positive cycle $Y \leqslant$ $Z_{0}$. Since $\cup\left\{E \mid E Z_{0}=0\right\}=\bigcup_{i=2}^{n-1} E_{i}$ is connected, $Y=Y_{1}$, where $Y_{1}$ is the fundamental cycle on $\bigcup_{i=2}^{n-1} E_{i}$; that is, $Y_{1}=\sum_{i=2}^{n-1} E_{i}$. Conversely,

$$
Z_{1}=Z_{0}+Y_{1}=\bigodot_{E_{1}}^{1}-\stackrel{2}{\bigcirc}-\stackrel{2}{\bigcirc}-\cdots \frac{2}{\bigcirc} \bigcirc \underset{E_{2}}{\bigcirc} \underset{E_{n-1}}{\bigcirc} \underset{E_{n}}{\bigcirc}
$$

is a special cycle by Theorem 4.10. Similarly, if we put $Y_{k}=\sum_{i=k+1}^{2 m-k} E_{i}$ for every $k=1,2, \ldots, m-1$, then we have:

(a) $0<Y_{m-1}<Y_{m-2}<\cdots Y_{2}<Y_{1} \leqslant Z_{0}$;

(b) $p_{a}\left(Y_{k}\right)=0$ and $Y_{k} Z_{k-1}=0$ for every $k=1, \ldots, m-1$;

(c) $Z_{k}=Z_{k-1}+Y_{k}$ is anti-nef for every $k=1, \ldots, m-1$;

(d) $\operatorname{coeff}_{E_{i}} Y_{k}=n_{i}$ if and only if $k+1 \leqslant i \leqslant 2 m-k$.

This produces a sequence of Ulrich ideals:

$$
I_{Z_{m-1}} \subset I_{Z_{m-2}} \subset \cdots \subset I_{Z_{1}}=\mathfrak{m} .
$$

We can determine Ulrich ideals in the case of $\left(A_{2 m+1}\right)$ similarly.

Finally, we consider the case $\left(D_{n}\right): f=x^{2}+x y^{n-3}+z^{2}$.

The case $\left(D_{2 m}\right): f=x^{2}+x y^{2 m-3}+z^{2}$. The fundamental cycle $Z_{0}$ on the minimal resolution of singularities is given by

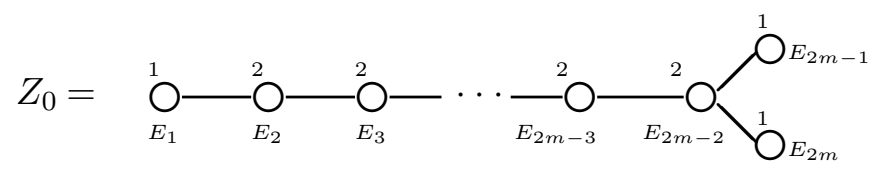

That is, $Z_{0}=E_{1}+2 \sum_{i=2}^{2 m-2} E_{i}+E_{2 m-1}+E_{2 m}$.

Now suppose that $Z_{0}+Y$ is a special cycle on $X$ for some positive cycle $Y \leqslant Z_{0}$. Since $\cup\left\{E \mid E Z_{0}=0\right\}$ has two connected components, we have that $Y=E_{1}$ or $Y=Y_{1}$ :

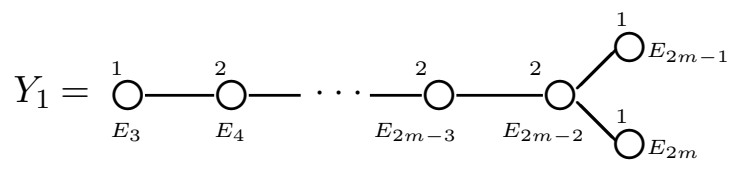

Conversely,

$$
Z_{1}^{\prime}=Z_{0}+E_{1}=\bigodot_{E_{1}}^{2}-\stackrel{2}{\bigcirc}-\stackrel{2}{\bigcirc}-\cdots \frac{2}{E_{2}}-\frac{2}{E_{2}} \overbrace{E_{2 m-2}}^{1} \overbrace{E_{2 m-1}}^{1}
$$


and

$$
Z_{1}=Z_{0}+Y_{1}=\bigcirc_{E_{1}}^{1}-\bigcirc_{E_{2}}^{2}-\bigcirc_{E_{3}}^{3}-\bigcirc_{E_{4}}^{4}-\cdots
$$

are special cycles by Theorem 4.10 .

Suppose that $Z_{1}+Y$ is a special cycle for some positive cycle $Y \leqslant Y_{1}$. Since $\cup\left\{E \subset \operatorname{Supp}\left(Y_{1}\right) \mid E Z_{1}=0\right\}$ has two connected components, we have $Y=E_{3}$ or $Y=Y_{2}$, where

$$
Y_{2}=\bigcirc_{E_{5}}^{1}
$$

Then $Z_{1}+E_{3}$ is not anti-nef, but

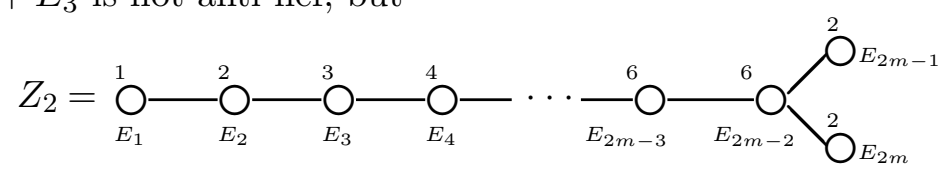

is a special cycle. Similarly, if we put

$$
Y_{k}=E_{2 k+1}+2 \sum_{i=2 k+2}^{2 m-2} E_{i}+E_{2 m-1}+E_{2 m}, \quad Z_{k}=Z_{k-1}+Y_{k},
$$

for each $k=1,2, \ldots, m-2$, then we have a sequence of positive cycles

$$
0<Y_{m-2}<Y_{m-3}<\cdots<Y_{1} \leqslant Z_{0} \text {. }
$$

By Theorem $4.10, Z_{0}, Z_{1}, \ldots, Z_{m-2}$ are special cycles. Note that

$$
\cup\left\{E \subset \operatorname{Supp}\left(Y_{m-2}\right) \mid E Z_{m-2}=0\right\}=E_{2 m-3} \cup E_{2 m-1} \cup E_{2 m}
$$

and

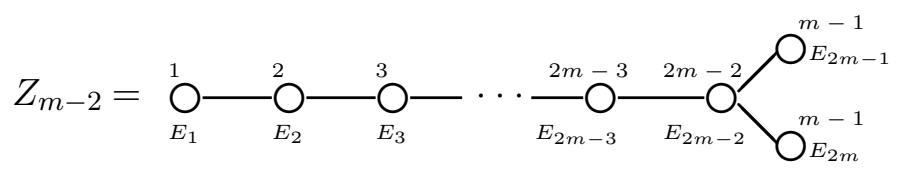

By a similar argument to that above, we obtain two minimal special cycles:

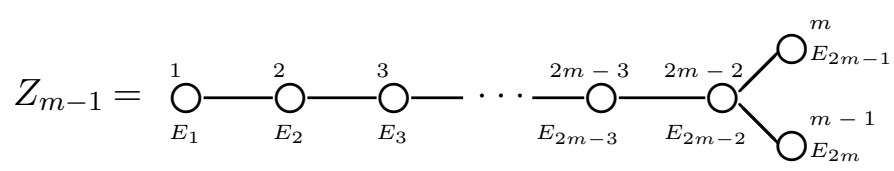




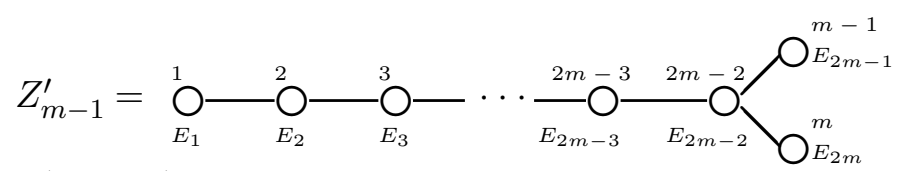

The case $\left(D_{2 m+1}\right)$. The fundamental cycle $Z_{0}$ on the minimal resolution is given by

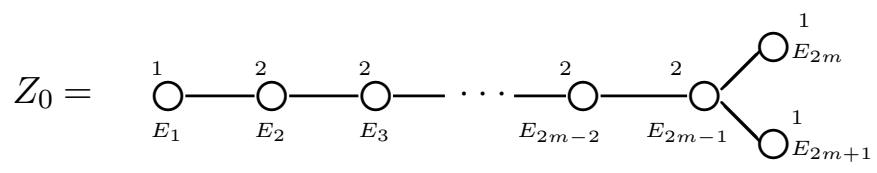

If we put

$$
\begin{gathered}
Y_{k}=E_{2 k+1}+2 \sum_{i=2 k+2}^{2 m-1} E_{i}+E_{2 m}+E_{2 m+1}, \\
Z_{k}=Z_{k-1}+Y_{k}=\sum_{i=1}^{2 k+1} i E_{i}+(2 k+2) \sum_{i=2 k+2}^{2 m-1} E_{i}+(k+1)\left(E_{2 m}+E_{2 m+1}\right),
\end{gathered}
$$

for each $k=1, \ldots, m-2$, then $0<Y_{m-2}<\cdots<Y_{2}<Y_{1} \leqslant Z_{0}$ are positive cycles and $Z_{0}, Z_{1}, \ldots, Z_{m-2}$ are special cycles.

Now suppose that $Z_{m-2}+Y$ is a special cycle for some positive cycle $Y \leqslant Y_{m-2}$. Since

$$
\cup\left\{E \subset \operatorname{Supp}\left(Y_{m-2}\right) \mid E Z_{m-2}=0\right\}=E_{2 m-1} \cup E_{2 m} \cup E_{2 m+1}
$$

is connected, we have that $Y=E_{2 m+1}+E_{2 m}+E_{2 m+1}$.

Set $Y_{m-1}=E_{2 m+1}+E_{2 m}+E_{2 m+1}$. Conversely, $Z_{m-1}=Z_{m-2}+Y_{m-1}$ is a special cycle by Theorem 4.10. Note that $Z_{m-1}$ is the minimal one among those special cycles.

\section{$\S 6$. Ulrich ideals of non-Gorenstein rational singularities}

In this section, we study Ulrich ideals of two-dimensional non-Gorenstein rational singularities. Notice that the maximal ideal $\mathfrak{m}$ is always an Ulrich ideal of such a local ring.

We first show that any Ulrich ideal of a two-dimensional rational singularity is a good ideal. In order to obtain a characterzation of Ulrich ideals, we need the following definition.

Throughout this section, let $(A, \mathfrak{m})$ be a two-dimensional non-Gorenstein rational singularity, and let $\varphi: X \rightarrow$ Spec $A$ be the minimal resolution of singularities. 
Definition 6.1. Let $\widetilde{\varphi}: \widetilde{X} \rightarrow$ Spec $A$ be a resolution of singularities of Spec $A$. Decompose $\widetilde{\varphi}$ as $\widetilde{\varphi}=\varphi \circ \pi$, where $\pi: \widetilde{X} \rightarrow X$. Let $\pi^{*} Z_{0}$ denote the pull-back of the fundamental cycle $Z_{0}$ on the minimal resoluition to $\widetilde{X}$. Then for any anti-nef cycle $Z$ on $\widetilde{X}$, we put

$$
U(Z)=\left(\varphi^{*} Z_{0} \cdot Z\right)\left(p_{a}(Z)-1\right)+Z^{2},
$$

where $p_{a}(Z)$ denotes the virtual genus of $Z$ (see the remark below).

Theorem 6.2. Let $(A, \mathfrak{m})$ be a two-dimensional rational singularity. Let $I$ be an $\mathfrak{m}$-primary ideal with $\mu_{A}(I)>2$. Then the following conditions are equivalent:

(1) $I$ is an Ulrich ideal;

(2) $\mathrm{e}_{I}^{0}(A)=(\mu(I)-1) \cdot \ell_{A}(A / I)$;

(3) $I$ is an integrally closed ideal represented on the minimal resolution of singularities $\varphi: X \rightarrow \operatorname{Spec} A$ such that $I \mathcal{O}_{X}=\mathcal{O}_{X}(-Z), I=$ $\mathrm{H}^{0}\left(X, \mathcal{O}_{X}(-Z)\right)$ and $U(Z)=0$.

Proof. (1) $\Longleftrightarrow(2)$ follows from [GOTWY, Lemma 2.3].

$(3) \Longrightarrow(2)$ : Any integrally closed ideal $I$ in a two-dimensional rational singularity is stable. Moreover, $U(Z)=0$ means that $\mathrm{e}_{I}^{0}(A)=(\mu(I)-1)$. $\ell_{A}(A / I)$. Thus, the assertion immediately follows from this.

$(2) \Longrightarrow(3)$ : Since $I$ is an Ulrich ideal by (1), we have that $I=Q: I$ for any minimal reduction $Q$ of $I$ by [GOTWY, Corollary 2.6]. Then as $\bar{I}^{2}=Q \bar{I}$, we get $I \subseteq \bar{I} \subseteq Q: \bar{I} \subseteq Q: I$. Hence, $I=\bar{I}$ is integrally closed.

Let $\tilde{\varphi}: \widetilde{X} \rightarrow$ Spec $A$ be a resolution of singularities so that $I=$ $\mathrm{H}^{0}\left(\widetilde{X}, \mathcal{O}_{\tilde{X}}(-Z)\right)$ and $I \mathcal{O}_{\tilde{X}}=\mathcal{O}_{\widetilde{X}}(-Z)$ is invertible for some anti-nef cycle $Z$ on $\tilde{X}$. Then (2) implies that $U(Z)=0$.

Now suppose that $I$ is not represented on the minimal resolution of singularities $\varphi: X \rightarrow \operatorname{Spec} A$. Then there exists a contraction $\psi: \widetilde{X} \rightarrow X^{\prime}$ of a $(-1)$-curve $E$ on $\widetilde{X}$ such that $I$ is not represented on $X^{\prime}$. Consider the following commutative diagram:

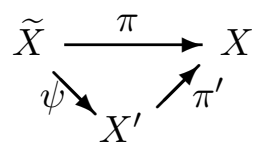

Then we may assume that $Z=\psi^{*} Z^{\prime}+n E$ for some anti-nef cycle $Z^{\prime}$ on $X^{\prime}$ and an integer $n \geqslant 1$. Note that $\pi^{*} Z_{0} \cdot E=\psi^{*} Z^{\prime} \cdot E=0$ (see, e.g., [GIW, Fact 7.7]). Then 


$$
\begin{aligned}
U(Z)-U\left(Z^{\prime}\right)= & \left(\pi^{*} Z_{0} \cdot\left(\psi^{*} Z^{\prime}+n E\right)\right)\left(p_{a}\left(\psi^{*} Z^{\prime}\right)+p_{a}(n E)+\psi^{*} Z^{\prime} \cdot n E-2\right) \\
& +\left(\psi^{*} Z^{\prime}+n E\right)^{2}-\left(\pi^{*} Z_{0} \cdot \psi^{*} Z^{\prime}\right)\left(p_{a}\left(\psi^{*} Z^{\prime}\right)-1\right)-\left(\psi^{*} Z^{\prime}\right)^{2} \\
= & \left(\pi^{*} Z_{0} \cdot \psi^{*} Z^{\prime}\right)\left(p_{a}(n E)-1\right)+(n E)^{2} \\
= & \left(\left(\pi^{\prime}\right)^{*} Z_{0} \cdot Z^{\prime}+2\right) \frac{(n E)^{2}}{2}+\frac{n\left(K_{X} \cdot E\right)}{2}\left(\left(\pi^{\prime}\right)^{*} Z_{0} \cdot Z^{\prime}\right) .
\end{aligned}
$$

Since $\left(\pi^{\prime}\right)^{*} Z_{0} \cdot Z^{\prime} \leqslant-2$ and $E^{2}=K_{X} \cdot E=-1$, we get $U(Z)>U\left(Z^{\prime}\right) \geqslant 0$. This is a contradiction.

In what follows, we always assume that $\varphi: X \rightarrow \operatorname{Spec} A$ is the minimal resolution of singularities, $I \mathcal{O}_{X}=\mathcal{O}_{X}(-Z)$ is invertible and $I=$ $\mathrm{H}^{0}\left(X, \mathcal{O}_{X}(-Z)\right)$ for some anti-nef cycle $Z$ on $X$. Let $\varphi^{-1}(\mathfrak{m})=\bigcup_{i} E_{i}$ denote the exceptional divisor on $X$ with the irreducible components $\left\{E_{i}\right\}_{1 \leqslant i \leqslant r}$. Let $Z_{0}$ (resp. $K$ ) denote the fundamental cycle (resp. the canonical divisor) on $X$. Notice that $Z_{0} E \leqslant 0$ and $K E=-E^{2}-2$ for all exceptional curves $E$.

The next target is to characterize Ulrich cycles in terms of dual graphs. In order to do that, we recall the sequence of anti-nef cycles introduced in Lemma 4.9. Assume that $Z \neq Z_{0}$ is an anti-nef cycle on $X$. Then we can find the following anti-nef cycles $Z_{1}, \ldots, Z_{s}$ and positive cycles $Y_{1}, \ldots, Y_{s}$, so that $0<Y_{s} \leqslant Y_{s-1} \leqslant \cdots \leqslant Y_{1} \leqslant Z_{0}$ :

$$
\left\{\begin{aligned}
Z=Z_{s} & =Z_{s-1}+Y_{s}, \\
Z_{s-1} & =Z_{s-2}+Y_{s-1}, \\
& \vdots \\
Z_{2} & =Z_{1}+Y_{2}, \\
Z_{1} & =Z_{0}+Y_{1} .
\end{aligned}\right.
$$

The following lemma plays a key role in the proof of the main theorem in this section.

Lemma 6.3. Let $Z, Z^{\prime}$ be anti-nef cycles on $X$ with $Z^{\prime}=Z+Y$, where $Y$ is a positive cycle. Then we have the following.

(1) $U\left(Z^{\prime}\right)-U(Z)=\left(Y Z_{0}\right)\left\{\left(p_{a}(Z)-1\right)+\left(p_{a}(Y)-1\right)\right\}+(Y Z)\left(Z^{\prime} Z_{0}+2\right)$ $+\left(p_{a}(Y)-1\right)\left(Z Z_{0}+2\right)-K Y$.

(2) Assume that $0 \neq Y \leqslant Z_{0}$ and $e=\mathrm{e}_{\mathfrak{m}}^{0}(A) \geqslant 3$. Then $U\left(Z^{\prime}\right) \geqslant U(Z)$ holds true, and equality holds if and only if $Y Z=Y Z_{0}=p_{a}(Y)=(Z-$ $\left.Z_{0}\right) Z_{0}=K\left(Z_{0}-Y\right)=0$. 
Proof. (1) Since $p_{a}(Z+Y)=p_{a}(Z)+p_{a}(Y)+Y Z-1$ by definition, we have

$$
\begin{aligned}
U\left(Z^{\prime}\right)-U(Z)= & \left(Z Z_{0}+Y Z_{0}\right)\left(p_{a}(Z)-1+p_{a}(Y)-1+Y Z\right) \\
& +\left(Z^{2}+2 Y Z+Y^{2}\right)-\left(Z Z_{0}\right)\left(p_{a}(Z)-1\right)-Z^{2} \\
= & \left(Y Z_{0}\right)\left\{\left(p_{a}(Z)-1\right)+\left(p_{a}(Y)-1\right)\right\} \\
& +(Y Z)\left(Z Z_{0}+Y Z_{0}+2\right)+\left(p_{a}(Y)-1\right)\left(Z Z_{0}\right)+Y^{2} \\
= & \left(Y Z_{0}\right)\left\{\left(p_{a}(Z)-1\right)+\left(p_{a}(Y)-1\right)\right\}+(Y Z)\left(Z^{\prime} Z_{0}+2\right) \\
& +\left(p_{a}(Y)-1\right)\left(Z Z_{0}+2\right)-K Y,
\end{aligned}
$$

where the last equality follows from $2\left(p_{a}(Y)-1\right)=K Y+Y^{2}$.

(2) Assume that $Y \leqslant Z_{0}$. As $X \rightarrow \operatorname{Spec} A$ is the minimal resolution, we have that $K Y \leqslant K Z_{0}$ because $K E \geqslant 0$ for all curves $E$ on $X$. Since $Z_{0}$ is anti-nef and $Z-Z_{0}, Y$ are positive, we get

$$
Z^{\prime} Z_{0}+2=\left(Z-Z_{0}\right) Z_{0}+Y Z_{0}+\left(Z_{0}^{2}+2\right) \leqslant Z_{0}^{2}+2=-e+2<0 .
$$

Moreover, $p_{a}\left(Z_{0}\right)=0$ implies that

$$
\begin{aligned}
& \left(p_{a}(Y)-1\right)\left(Z Z_{0}+2\right)-K Y \\
& \quad=p_{a}(Y)\left(Z Z_{0}+2\right)-\left(Z-Z_{0}\right) Z_{0}-K\left(Y-Z_{0}\right) \geqslant 0,
\end{aligned}
$$

and equality holds if and only if $p_{a}(Y)=\left(Z-Z_{0}\right) Z_{0}=K\left(Y-Z_{0}\right)=0$.

Note that $Y Z_{0}, Y Z \leqslant 0$ and $p_{a}(Z)-1+p_{a}(Y)-1<0$. Hence, $U\left(Z^{\prime}\right) \geqslant$ $U(Z)$, and equality holds if and only if $Y Z_{0}=Y Z=0$ and $p_{a}(Y)=(Z-$ $\left.Z_{0}\right) Z_{0}=K\left(Y-Z_{0}\right)=0$.

The main result in this section is the following theorem, which enables us to determine all Ulrich ideals of a two-dimensional (non-Gorenstein) rational singularity. For a positive cycle $Z$ on $X$, we write $Z=\sum_{E} Z_{E} E$, where $Z_{E}$ is a nonnegative integer.

TheOrem 6.4. Let $(A, \mathfrak{m})$ be a two-dimensional rational singularity with $e=\mathrm{e}_{\mathfrak{m}}^{0}(A) \geqslant 3$, and let $\varphi: X \rightarrow \operatorname{Spec} A$ be the minimal resolution of singularities. Set $Z_{0}=\sum_{E} n_{E} E$, the fundamental cycle on $X$. Let $Z$ be an anti-nef cycle on $X$ with $I \mathcal{O}_{X}=\mathcal{O}_{X}(-Z)$ and $I=\mathrm{H}^{0}\left(X, \mathcal{O}_{X}(-Z)\right)$. Then the following conditions are equivalent:

(1) I is an Ulrich ideal, that is, $Z$ is an Ulrich cycle on $X$; 
(2) there exist a sequence of anti-nef cycles $Z_{1}, \ldots, Z_{s}$ and a sequence of positive cycles $0<Y_{s} \leqslant \cdots \leqslant Y_{1} \leqslant Z_{0}$ for some $s \geqslant 1$ so that

$$
\left\{\begin{aligned}
Z=Z_{s} & =Z_{s-1}+Y_{s}, \\
Z_{s-1} & =Z_{s-2}+Y_{s-1}, \\
& \vdots \\
Z_{1} & =Z_{0}+Y_{1},
\end{aligned}\right.
$$

and $Y_{k} Z_{k-1}=p_{a}\left(Y_{k}\right)=K\left(Z_{0}-Y_{k}\right)=0$ for every $k=1, \ldots, s$.

When this is the case, the following conditions are satisfied.

(a) $\left\{E \mid E^{2} \leqslant-3\right\}$ is contained in $\operatorname{Supp}\left(Y_{1}\right)$.

(b) $\operatorname{Supp}\left(Y_{k}\right)$ is given as one of the connected components of $\left\{E \mid E Z_{k-1}=\right.$ $0\}$ in $\left\{E \mid E Z_{0}=0\right\}$.

(c) $Y_{k}$ is the fundamental cycle on $\operatorname{Supp}\left(Y_{k}\right)$.

(d) $\operatorname{coeff}_{E} Z_{0}=\operatorname{coeff}_{E} Y_{k}$ for every $E$ with $E^{2} \leqslant-3$.

If, in addition, we put $I_{k}=\mathrm{H}^{0}\left(X, \mathcal{O}_{X}\left(-Z_{k}\right)\right)$, then $I_{k}$ is an Ulrich ideal, so that

$$
\mathfrak{m}=I_{0} \supseteq I_{1} \supseteq \cdots \supseteq I_{s}=I \quad \text { and } \quad \ell_{A}(A / I)=s+1 .
$$

Proof. Take a sequence as in (2).

$(1) \Longrightarrow(2)$ : Lemma 6.3 implies that

$$
0=U(Z)=U\left(Z_{s}\right) \geqslant U\left(Z_{s-1}\right) \geqslant \cdots \geqslant U\left(Z_{1}\right) \geqslant U\left(Z_{0}\right)=0 .
$$

Hence, all $Z_{k}$ are Ulrich cycles and

$$
Y_{k} Z_{k-1}=Y_{k} Z_{0}=p_{a}\left(Y_{k}\right)=\left(Z_{k}-Z_{0}\right) Z_{0}=K\left(Z_{0}-Y_{k}\right)=0
$$

for every $k=1, \ldots, s$. By a similar argument to that in the proof of Theorem 4.10, we have $\ell_{A}(A / I)=s+1$.

If $E^{2} \leqslant-3$, then $K E=-E^{2}-2>0$. Thus, $K\left(Z_{0}-Y_{1}\right)=0$ implies that coeff $_{E} Z_{0}=$ coeff $_{E} Y_{1}$ for every $E$ with $E^{2} \leqslant-3$. In particular, $\operatorname{Supp}\left(Y_{k}\right) \supseteq$ $\left\{E \mid E^{2} \leqslant-3\right\}$. On the other hand, $Y_{k} Z_{0}=0$ implies that $\operatorname{Supp}\left(Y_{i}\right) \subseteq\{E \mid$ $\left.E Z_{0}=0\right\}$ because $Z_{0}$ is an anti-nef cycle.

Now suppose $(2)$. Fix $i$ with $1 \leqslant k \leqslant r$. Since $Z_{k-1}$ is anti-nef and $Y_{k} Z_{k-1}=0$, a similar argument to the above yields that $\left\{E \mid E^{2} \leqslant-3\right\} \subseteq$ $\operatorname{Supp}\left(Y_{k}\right) \subseteq\left\{E \mid E Z_{k-1}=0\right\}$.

As $p_{a}\left(Y_{k}\right)=0, \operatorname{Supp}\left(Y_{k}\right)$ is connected. Moreover, $\operatorname{Supp}\left(Y_{k}\right)$ is one of the connected components of $\left\{E \mid E Z_{k-1}=0\right\}$. Indeed, if there exists a curve 
$E \notin \operatorname{Supp}\left(Y_{k}\right)$ such that $E E^{\prime}>0$ for some $E^{\prime} \in \operatorname{Supp}\left(Y_{k}\right)$, then $E Z_{k-1}<0$ since $E Y_{k} \geqslant 1$ and $E Z_{k-1}+E Y_{k}=E Z_{k} \leqslant 0$.

Claim. $Y_{k}$ is the fundamental cycle on $\operatorname{Supp}\left(Y_{k}\right)$.

Take $E \in \operatorname{Supp}\left(Y_{k}\right)$. As $Y_{k} Z_{k-1}=0$, we have $E Z_{k-1}=0$. If $E Y_{k}>0$, then $E Y_{k}=E Z_{k} \leqslant 0$. This is a contradiction. Hence, $E Y_{k} \leqslant 0$. Namely, $Y_{k}$ is antinef. Moreover, if $Y_{k}$ is not the fundamental cycle on $\operatorname{Supp}\left(Y_{k}\right)$, then we know that $p_{a}\left(Y_{k}\right) \leqslant-1$. This contradicts the assumption $p_{a}\left(Y_{k}\right)=0$. Hence, $Y_{k}$ must be the fundamental cycle on $\operatorname{Supp}\left(Y_{k}\right)$.

To see $(2) \Longrightarrow(1)$, we notice that (c) implies that $p_{a}\left(Y_{k}\right)=0$. Condition (b) means that $Y_{k} Z_{k-1}=0$. Hence, $Y_{k} Z_{0}=0$. Note that the equalities $Y_{k} Z_{0}=Y_{k-1} Z_{0}=\cdots=Y_{1} Z_{0}=0$ yield $\left(Z_{k}-Z_{0}\right) Z_{0}=0$. Condition (d) implies that $K\left(Z_{0}-Y_{k}\right)=0$. Therefore, $U(Z)=U\left(Z_{r}\right)=\cdots=U\left(Z_{1}\right)=$ $U\left(Z_{0}\right)=0$, as required.

The following assertion does not hold true without the assumption that $A$ is rational (see [GOTWY, Example 2.2]).

Corollary 6.5. Let $A$ be a two-dimensional rational singularity. If I is an Ulrich ideal of $A$, then $I$ is a special ideal and $A / I$ is Gorenstein.

Proof. Denote by $\mathfrak{m}$ the maximal ideal of $R$. We may assume that $A$ is not Gorenstein, that is, $e=\mathrm{e}_{\mathfrak{m}}^{0}(A) \geqslant 3$. Then by Theorem 6.4 , we can find a sequence of Ulrich cycles $Z_{1}, \ldots, Z_{s}$ and positive cycles $0<Y_{1} \leqslant \cdots \leqslant Y_{s} \leqslant$ $Z_{0}$ satisfying all conditions in Theorem 6.4 , so that

$$
\left\{\begin{aligned}
Z=Z_{s} & =Z_{s-1}+Y_{s} \\
Z_{s-1} & =Z_{s-2}+Y_{s-1} \\
& \vdots \\
Z_{1} & =Z_{0}+Y_{1}
\end{aligned}\right.
$$

Then $Z \leqslant(s+1) Z_{0}$ and $Z \not s Z_{0}$. In particular, $\mathfrak{m}^{s} \nsubseteq \nsubseteq I$ and $\mathfrak{m}^{s+1} \subseteq I$. Moreover, $I$ is a special ideal by Theorem 4.10 . We have only to show the following claim.

Claim. There exists a minimal set of generators $\left\{u_{1}, \ldots, u_{p}, t\right\}$ such that $I=\left(u_{1}, \ldots, u_{p}, t^{s+1}\right)$.

Set $I_{s-1}=\mathrm{H}^{0}\left(X, \mathcal{O}_{X}\left(-Z_{s-1}\right)\right)$. Then $I_{s-1}$ is also an Ulrich ideal. Therefore, we may assume that we can write $I_{s-1}=\left(u_{1}, \ldots, u_{p}, t^{s}\right)$ for some minimal set of generators of $\mathfrak{m}$. Since $\mathfrak{m}\left(u_{1}, \ldots, u_{p}\right) \subseteq I$ and $\mathfrak{m}^{s} \nsubseteq I$, we 
have that $t^{s} \notin I$. Hence, by $\ell_{A}\left(I_{s-1} / I\right)=1$, we can choose an element $a_{i} \in A$ such that $u_{i}-a_{i} t^{s} \in I$ for every $i$. By replacing $u_{i}$ with $u_{i}-a_{i} t^{s}$, we may assume that $I^{\prime}=\left(u_{1}, \ldots, u_{p}, t^{s+1}\right) \subseteq I$. As $\ell_{A}\left(I_{s-1} / I^{\prime}\right)=1$ and $I \neq I_{s-1}$, we can conclude that $I=I^{\prime}$, as required.

\section{$\S 7 . \quad$ Examples}

Throughout this section, let $k$ be an algebraically closed field of characteristic 0 . Let $\mathcal{X}_{A}$ denote the set of nonparameter Ulrich ideals of $A$.

ExAmPLE 7.1. Let $A=k[[x, y, z]] /\left(x^{2}+y^{4}+z^{2}\right) \cong k\left[\left[s^{4}, s t, t^{4}\right]\right]$ be a two-dimensional rational double point of type $\left(A_{4}\right)$. Theorem 5.7 implies that $\mathcal{X}_{A}=\left\{\mathfrak{m}, I_{1}\right\}$, where $\mathfrak{m}=(x, y, z)=\left(s^{4}, s t, t^{4}\right)$ and $I_{1}=\left(x, y^{2}, z\right)=$ $\left(s^{4}, s^{2} t^{2}, t^{4}\right)$.

The corresponding anti-nef cycle to $\mathfrak{m}$ (resp. $I_{1}$ ) on the minimal resolution is

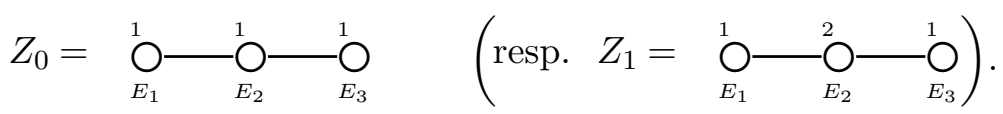

Moreover, if we put

$$
M_{0}=A, \quad M_{1}=A s+A t^{3}, \quad M_{2}=A s^{2}+A t^{2} \quad \text { and } \quad M_{3}=A s^{3}+A t,
$$

then they are representatives of indecomposable maximal Cohen-Macaulay $A$-modules, and thus any maximal Cohen-Macaulay $A$-module can be written as

$$
A^{\oplus k} \oplus M_{1}^{\oplus k_{1}} \oplus M_{2}^{\oplus k_{2}} \oplus M_{3}^{\oplus k_{3}}
$$

Note that $\operatorname{Syz}_{A}^{2}(A / \mathfrak{m}) \cong M_{1} \oplus M_{3}$ and $\operatorname{Syz}_{A}^{2}\left(A / I_{1}\right) \cong M_{2}^{\oplus 2}$. Moreover, Theorem 5.7 says that any Ulrich $A$-module with respect to $\mathfrak{m}$ (resp. $I_{1}$ ) can be written as

$$
M_{1}^{\oplus k_{1}} \oplus M_{2}^{\oplus k_{2}} \oplus M_{3}^{\oplus k_{3}} \quad \text { (resp. } M_{2}^{\oplus k_{2}} \text { ). }
$$

In general, there exists an Ulrich $A$-module with respect to $I$, but not a direct summand of $\operatorname{Syz}_{A}^{i}(A / I)$, which contradicts Example 7.1.

ExAmple 7.2. Let $A=k[[x, y, z]] /\left(x^{3}+y^{5}+z^{2}\right)$ be a rational double point of type $\left(E_{8}\right)$. By Theorem 5.7 and Corollary 5.8, the set of Ulrich 
ideals is $\mathcal{X}_{A}=\{\mathfrak{m}, I\}$, where $I=\left(x, y^{2}, z\right)$ with $\ell_{A}(A / I)=2$.

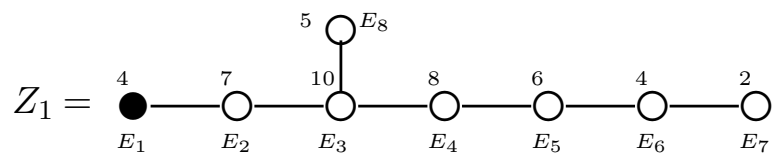

Let $M_{i}$ denote the indecomposable maximal Cohen-Macaulay $A$-module corresponding to $E_{i}$ (up to equivalence) via the McKay correspondence for every $i=1, \ldots, 8$. Then we have $\operatorname{Syz}_{A}^{2}(A / I) \cong M_{1}$. Indeed, since $\operatorname{Syz}_{A}^{2}(A / I)$ is an Ulrich $A$-module with respect to $I$, it is isomorphic to $M_{1}^{\oplus k}$ for some $k \geqslant 1$. Then $k=1$ because $\operatorname{rank} \operatorname{Syz}_{A}^{2}(A / I)=\operatorname{rank} M_{1}=2$.

Next we see that $\operatorname{Syz}_{A}^{2}(A / \mathfrak{m}) \cong M_{7}$. Set $\Omega=\operatorname{Syz}_{A}^{2}(A / \mathfrak{m}) . \operatorname{As}_{\operatorname{rank}_{A}} \Omega=2$, we have $\Omega \cong M_{1}$ or $\Omega \cong M_{7}$. It follows from [GOTWY, Corollary 7.7] that $\Omega \cong M_{7}$.

Similarly, one can easily see that $\operatorname{Syz}_{A}^{i}(A / \mathfrak{m}) \cong M_{7}$ and $\operatorname{Syz}_{A}^{i}(A / I) \cong M_{1}$ for every $i \geqslant 2$. Hence, $M_{2}$ cannot be written as a direct summand of $\operatorname{Syz}_{A}^{i}(A / J)$ for any Ulrich ideal $J$.

Two-dimensional rational double points $\left(A_{n}\right)$ (Gorenstein quotient singularities) admit a sequence of Ulrich ideals of length $m=\left\lceil\frac{n}{2}\right\rceil$ :

$$
\left(x, y^{m}, z\right) \subset \cdots \subset\left(x, y^{2}, z\right) \subset(x, y, z) .
$$

However, the following example shows that each two-dimensional nonGorenstein cyclic quotient singularity has a unique Ulrich ideal (that is, the maximal ideal $\mathfrak{m}$ ).

ExAmPle 7.3. (Cyclic quotient singularity) Let $A$ be a two-dimensional cyclic quotient singularity of type $\frac{1}{n}(1, q)$, where $q$ and $n$ are integers with $1<q<n,(q, n)=1$. Namely, $A$ is the invariant subring of the cyclic group generated by

$$
g=\left[\begin{array}{cc}
\varepsilon_{n} & 0 \\
0 & \varepsilon_{n}^{q}
\end{array}\right]
$$

where $\varepsilon_{n}$ denotes the primitive $n$th root of $1 \in k$.

Now suppose that $A$ is not Gorenstein, that is, $q+1$ is not divided by $n$. Then there exists an exceptional curve $E_{i}$ so that $b:=-E_{i}^{2} \geqslant 3$. In particular, $K E_{i}=b-2 \geqslant 1$. Let $\Gamma$ be the dual graph of the minimal 
resolution of singularities $X \rightarrow \operatorname{Spec} A$.

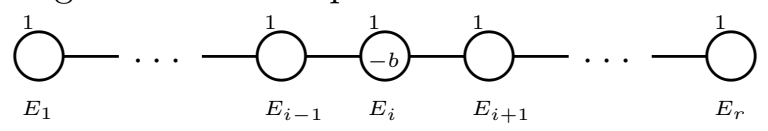

It is well known that $\mathfrak{m}$ is an Ulrich ideal. Now suppose that there exists an Ulrich ideal other than $\mathfrak{m}$. Then we can take $Y_{1}$ satisfying the conditions (a), (b) in Theorem 6.4. In particular, $Z_{0} Y_{1}=0, K\left(Z_{0}-Y_{1}\right)=0$ and $0<$ $Y_{1} \leqslant Z_{0}$. Set $Y_{1}=\sum_{j \in J} E_{j}$ for some non-empty subset $J$ of $\{1, \ldots, r\}$.

If $i \in J$, then $Z_{0} E_{i}=0$ because $Z_{0} Y_{1}=0$. On the other hand, $Z_{0} E_{i} \leqslant E_{i}^{2}+2=2-b \leqslant-1$. This is a contradiction. Hence, $i \notin J$. Then $E_{i} \subset \operatorname{Supp}\left(Z_{0}-Y_{1}\right)$. This implies that $K E_{i}=0$ because $K\left(Z_{0}-Y_{1}\right)=0$, which contradicts the choice of $E_{i}$. Hence, the maximal ideal is the only Ulrich ideal of $A$.

Remark 7.4. Let $A$ be a cyclic quotient singularity as above. Then one can obtain many examples of special cycles in general by a similar argument to the proof of Theorem 5.7.

EXAmPLE 7.5. (Rational triple points) Let $a \geqslant b \geqslant c \geqslant 2$. If we set $A=$ $k\left[\left[T, s T^{a}, s^{-1} T^{b},(s+1)^{-1} T^{c}\right]\right]$, then it is a two-dimensional rational singularity with $\mathrm{e}_{\mathfrak{m}}^{0}(A)=3$ and

$$
\begin{aligned}
A & \cong k[[t, x, y, z]] /\left(x y-t^{a+b}, x z-t^{a+c}+z t^{a}, y z-y t^{c}+z t^{b}\right) \\
& \cong k[[t, x, y, z]] / I_{2}\left(\begin{array}{cc}
x & t^{b} t^{c}-z \\
t^{a} y & z
\end{array}\right) .
\end{aligned}
$$

See [Wah, 3.6] and the dual graph of the minimal resolution $X \rightarrow \operatorname{Spec} A$ below.

Put $I_{k}=\left(t^{k}, x, y, z\right)$. Then $I_{k}$ is an Ulrich ideal of colength $k$ for every $k$ with $1 \leqslant k \leqslant c$. In fact, if we put $Q_{k}=\left(t^{k}, x+y+z\right)$, then $I_{k}=Q_{k}+(x, y)$ and $I_{k}^{2}=Q_{k} I_{k}$. Furthermore, we have $e_{I_{k}}^{0}(A)=\ell_{A}\left(A / Q_{k}\right)=3 k=(\mu(I)-$ $1) \cdot \ell_{A}(A / I)$. Hence, $I_{k}$ is an Ulrich ideal.

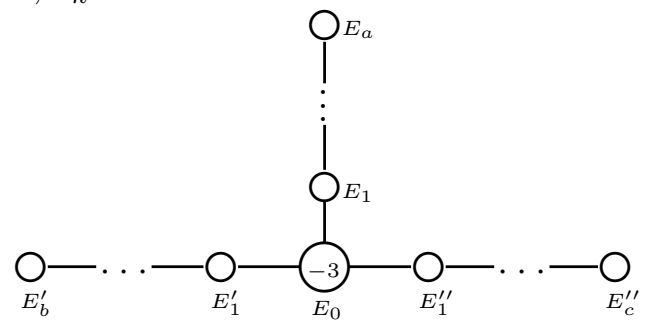


Let $a=b=c=3$. Now consider the corresponding cycles.
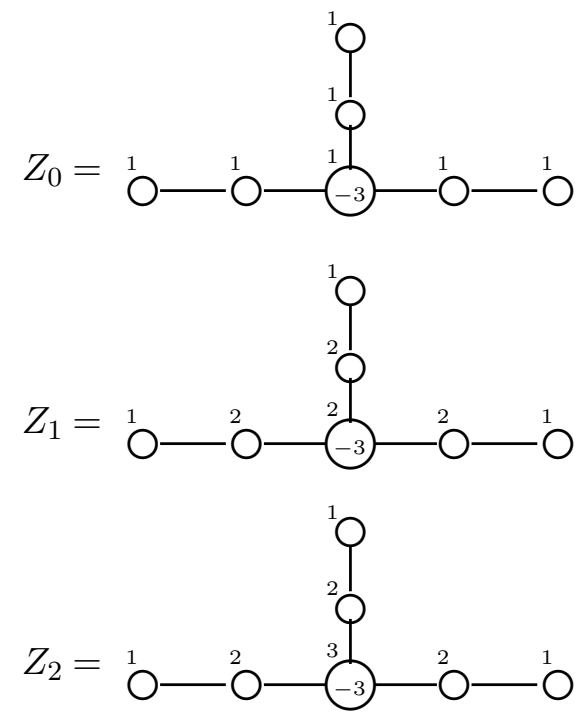

Note that all special cycles are Ulrich cycles.

Example 7.6. Let $A=k\left[\left[s^{7}, s^{4} t, s t^{2}, t^{7}\right]\right]$. Then $A$ is a two-dimensional cyclic quotient singularity, which is an invariant subring of a cyclic group generated by

$$
g=\left(\begin{array}{ll}
\varepsilon_{7} & \\
& \varepsilon_{7}^{3}
\end{array}\right)
$$

Since $\frac{7}{3}=3-\frac{1}{2-1 / 2}$, the dual graph can be written as the following form (e.g. $[\mathrm{Ri}])$ :

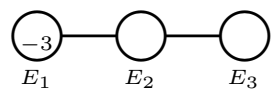

If we put $N_{a}=\left\langle s^{i} t^{j} \mid i+3 j \equiv a(\bmod 3)\right\rangle$ for $a=0,1, \ldots, 6$, then $\left\{N_{a}\right\}_{a=0}^{6}$ forms a representative of isomorphism classes of indecomposable maximal Cohen-Macaulay $A$-modules. Then $M_{1}=N_{3}=A s+A t^{5}, M_{2}=N_{2}=A s^{2}+$ $A t^{3}$ and $M_{3}=N_{1}=A s^{3}+A t$ are indecomposable special Cohen-Macaulay $A$-modules. On the other hand, $N_{4}=A s^{4}+A s t+A t^{6}, N_{5}=A s^{5}+A s^{2} t+$ $A t^{4}, N_{6}=A s^{6}+A s^{3} t+A t^{2}$ are indecomposable Ulrich $A$-modules with respect to the maximal ideal $\mathfrak{m}$. See also [Wu2]. 
All special cycles are $Z_{0}=E_{1}+E_{2}+E_{3}$ and $Z_{1}=E_{1}+2 E_{2}+E_{3}$. (Note that $Z_{1}$ is not an Ulrich cycle (see Example 7.3).) Any special CohenMacaulay module with respect to $I_{Z_{1}}$ is of the form $M_{2}^{\oplus k}$.

However, we do not have the complete list of Ulrich modules with respect to some ideal.

Acknowledgements. The authors thank Yukari Ito, Osamu Iyama, Yusuke Nakajima and Michael Wemyss for valuable comments and conversations. The authors are also grateful to the referee for reading the paper carefully and giving many valuable suggestions. In particular, Remark 4.7 is due to his/her advice.

\section{REFERENCES}

[Ab] S. S. Abhyankar, Local rings of high embedding dimension, Amer. J. Math. 89 (1967), 1073-1077.

[Ar] M. Artin, On isolated rational singularities of surfaces, Amer. J. Math. 88 (1966), 129-136.

[BHU] J. Brennan, J. Herzog and B. Ulrich, Maximally generated Cohen-Macaulay modules, Math. Scand. 61 (1987), 181-203.

[GIW] S. Goto, S. Iai and K. Watanabe, Good ideals in Gorenstein local rings, Trans. Amer. Math. Soc. 353 (2000), 2309-2346.

[GOTWY] S. Goto, K. Ozeki, R. Takahashi, K.-i. Watanabe and K.-i. Yoshida, Ulrich ideals and modules, Math. Proc. Cambridge Philos. Soc. 156 (2014), 133-166.

[HKuh] J. Herzog and M. Kühl, "Maximal Cohen-Macaulay modules over Gorenstein rings and Bourbaki-sequences", in Commutative Algebra and Combinatorics (Kyoto, 1985), Adv. Stud. Pure Math. 11, North-Holland, Amsterdam, 1987, 65-92.

[Ito] Y. Ito, Special McKay correspondence, Sémin. Congr. 6 (2002), 213-225.

[IW] O. Iyama and M. Wemyss, The classification of special Cohen-Macaulay modules, Math. Z. 265 (2010), 41-83.

[Ka] M. Kato, Riemann-Roch theorem for strongly convex manifolds of dimension 2, Math. Ann. 222 (1976), 243-250.

[Li1] J. Lipman, Rational singularities, with applications to algebraic surfaces and unique factorization, Publ. Math. Inst. Hautes Études Sci. 36 (1969), 195-279.

[Li2] J. Lipman, Desingularization of two-dimensional schemes, Ann. of Math. (2) 107 (1978), 151-207.

[Ma] H. Matsumura, Commutative Ring Theory, Cambridge Studies in Advanced Mathematics 8, Cambridge University Press, Cambridge, 1986.

[Na] Y. Nakajima, Dual F-signature of Cohen-Macaulay modules over rational double points, preprint, arXiv:1407.5230v2.

[Ri] O. Riemenschneider, Die Invarianten der endlichen Untergruppen von GL(2, C), Math. Z. 193 (1977), 37-50.

[Sa] J. Sally, Cohen-Macaulay local rings of maximal embedding dimension, J. Algebra 56 (1979), 168-183.

[U] B. Ulrich, Gorenstein rings and modules with high numbers of generators, Math. Z. 188 (1984), 23-32. 
[Vi] E. Viehweg, Rational singularities of higher dimensional schemes, Proc. Amer. Math. Soc. 63 (1977), 6-8.

[Wah] J. M. Wahl, Equations defining rational singularities, Ann. Sci. Éc. Norm. Supér. (4) 10 (1977), 231-263.

[WY] K.-I. Watanabe and K. Yoshida, Hilbert-Kunz multiplicity, McKay correspondence and good ideals in two-dimensional rational singularities, Manuscripta Math. 104 (2001), 275-294.

[Wu1] J. Wunram, Reflexive modules on quotient surface singularities, Math. Ann. 279 (1988), 583-598.

[Wu2] J. Wunram, Reflexive Modules on Cyclic Quotient Surface Singularities, Lecture Notes in Mathematics 1273, Springer, Berlin, 1987, 271-291.

[Y] Y. Yoshino, Cohen-Macaulay modules over Cohen-Macaulay rings, London Mathematical Society, Lecture Note Series 146, Cambridge University Press, Cambridge, 1990.

\section{Shiro Goto}

Department of Mathematics

School of Science and Technology

Meiji University

1-1-1 Higashimita

Tama-ku

Kawasaki 214-8571

Japan

goto@math.meiji.ac.jp

Kazuho Ozeki

Department of Mathematical Sciences

Faculty of Science

Yamaguchi University

1677-1 Yoshida

Yamaguchi 753-8512

Japan

ozeki@yamaguchi-u.ac.jp

Ryo Takahashi

Graduate School of Mathematics

Nagoya University

Furocho

Chikusaku

Nagoya 464-8602

Japan

takahashi@math.nagoya-u.ac.jp 
Kei-ichi Watanabe

Department of Matheamatics

College of Humanities and Sciences

Nihon University

3-25-40 Sakurajosui

Setagaya-Ku

Tokyo 156-8550

Japan

watanabe@math.chs.nihon-u.ac.jp

Ken-ichi Yoshida

Department of Matheamatics

College of Humanities and Sciences

Nihon University

3-25-40 Sakurajosui

Setagaya-Ku

Tokyo 156-8550

Japan

yoshida@math.chs.nihon-u.ac.jp 